\title{
Accelerated front propagation for monostable equations with nonlocal diffusion: Multidimensional case ${ }^{1}$
}

\author{
Dmitri Finkelshtein $^{2} \quad$ Yuri Kondratiev $^{3} \quad$ Pasha Tkachov $^{4}$
}

October 1, 2019

\begin{abstract}
We describe acceleration of the front propagation for solutions to a class of monostable nonlinear equations with a nonlocal diffusion in $\mathbb{R}^{d}$, $d \geq 1$. We show that the acceleration takes place if either the diffusion kernel or the initial condition has 'regular' heavy tails in $\mathbb{R}^{d}$ (in particular, decays slower than exponentially). Under general assumptions which can be verified for particular models, we present sharp estimates for the timespace zone which separates the region of convergence to the unstable zero solution with the region of convergence to the stable positive constant solution. We show the variety of different possible rates of the propagation starting from a little bit faster than a linear one up to the exponential rate. The paper generalizes to the case $d>1$ our results for the case $d=1$ obtained early in [31].
\end{abstract}

Keywords: nonlocal diffusion, reaction-diffusion equation, front propagation, acceleration, monostable equation, nonlocal nonlinearity, longtime behavior, integral equation

2010 Mathematics Subject Classification: 35B40, 35K57, 47G20, $45 \mathrm{G} 10$

\section{Contents}

1 Introduction $\mathbf{2}$

1.1 Object of study . . . . . . . . . . . . . . . . . . 2

1.2 Description of results . . . . . . . . . . . . . . . . . . 3

1.3 Overview of literature . . . . . . . . . . . . . 7

${ }^{1}$ Ethical Statement: Compliance with Ethical Standards. Funding: "No." Conflict of Interest: "The authors declare that they have no conflict of interest." Ethical approval: "This article does not contain any studies with human participants or animals performed by any of the authors." Informed consent: "Informed consent was obtained from all individual participants included in the study."

${ }^{2}$ Department of Mathematics, Swansea University, Singleton Park, Swansea SA2 8PP, U.K. (d.l.finkelshtein@swansea.ac.uk).

${ }^{3}$ Fakultät für Mathematik, Universität Bielefeld, Postfach 110 131, 33501 Bielefeld, Germany (kondrat@math. uni-bielefeld.de).

${ }^{4}$ Gran Sasso Science Institute, Viale Francesco Crispi, 7, 67100 L'Aquila AQ, Italy (pasha.tkachov@gssi.it). 
2 Assumptions and general results 9

2.1 General assumptions . . . . . . . . . . . . . . . . 9 9

2.2 General results . . . . . . . . . . . . . . . . . 12

3 Scheme of the proof $\quad \mathbf{1 5}$

3.1 Upper estimates . . . . . . . . . . . . . . . . . 15

3.2 Lower estimates . . . . . . . . . . . . . . . 17

4 Technical tools $\quad 19$

4.1 Functions constructed by tail-decreasing functions . . . . . . . . 19

4.2 Functions constructed by long-tailed functions . . . . . . . . . . 23

5 Proofs $\quad 24$

5.1 Proofs of upper estimates . . . . . . . . . . . . . . . . . . . 25

5.2 Proofs of lower estimates . . . . . . . . . . . . . . . . . 29

5.3 Proofs of general results . . . . . . . . . . . . . . 36

A Appendix

\section{Introduction}

\subsection{Object of study}

The present paper is aimed to study the accelerated propagation of the front for a non-negative solutions $u: \mathbb{R}^{d} \times \mathbb{R}_{+} \rightarrow \mathbb{R}_{+}:=[0, \infty), d \geq 1$, to the reactiondiffusion equation

$$
\frac{\partial}{\partial t} u(x, t)=(L u)(x, t)+(F u)(x, t)
$$

with an initial condition $u(x, 0)=u_{0}(x)$ in the space $E:=L^{\infty}\left(\mathbb{R}^{d}, d x\right)$. Here

$$
(L u)(x, t):=\int_{\mathbb{R}} a(x-y)(u(y, t)-u(x, t)) d y
$$

is the generator of a nonlocal diffusion in $\mathbb{R}^{d}$ (see e.g. $[3,11,33,44]$ ) with an (essentially) bounded probability kernel $a$ on $\mathbb{R}^{d}$, i.e.

$$
0 \leq a \in L^{1}\left(\mathbb{R}^{d}, d x\right) \cap E, \quad \int_{\mathbb{R}^{d}} a(x) d x=1
$$

and $F: E \rightarrow E$ describes a reaction (possibly also nonlocal). The solution $u$ to the equation (1.1) may be interpreted as a density of a species which invades according to a nonlocal diffusion within the space $\mathbb{R}^{d}$ meeting a reaction $F$, see e.g. $[25,51]$. By a solution to $(1.1)$ on $\mathbb{R}_{+}$, we understand the so-called classical solution, that is a mapping $u: \mathbb{R}_{+} \rightarrow E$ which is continuous in $t \in \mathbb{R}_{+}$and continuously differentiable (in the sense of the norm in $E$ ) in $t \in(0, \infty)$.

We consider a subclass of monostable reactions, see e.g. [6]. In particular, we assume that $F 0=F 1=0$, i.e. $u \equiv 0$ and $u \equiv 1$ are stationary solutions to (1.1), and also that

(i) there are not constant stationary solutions to (1.1) between 0 and 1 ; 
(ii) $u \equiv 0$ is an asymptotically unstable solution to (1.1), whereas $u \equiv 1$ is an asymptotically stable one;

(iii) for a given $u_{0} \in E$ with $0 \leq u_{0} \leq 1$, there exists a unique solution to (1.1) such that

$$
0 \leq u(x, t) \leq 1, \quad x \in \mathbb{R}^{d}, t>0
$$

Henceforth we write $v \leq w$ for $v, w \in E$, if $v(x) \leq w(x)$ for almost all (a.a. in the sequel) $x \in \mathbb{R}^{d}$. Moreover, we often just write $x \in \mathbb{R}^{d}$ omitting 'for a.a.' before this.

We will work under assumptions which ensure also that the solution $u$ satisfies the comparison principle (see Section 2 for details).

A typical example of the reactions we cover is given, for a fixed $\beta>0$, by

$$
(F u)(x)=\alpha f(u(x))+(1-\alpha) \beta u(x)\left(1-\int_{\mathbb{R}^{d}} a^{-}(x-y) u(y) d y\right)^{k},
$$

for $u \in E$. Here $\alpha \in[0,1], f: \mathbb{R} \rightarrow \mathbb{R}, k \in \mathbb{N}, 0 \leq a^{-} \in L^{1}(\mathbb{R}), \int_{\mathbb{R}} a^{-}(x) d x=1$ and we assume that

$$
\begin{gathered}
\frac{f(r)}{r} \text { is Lipschitz continuous on } r \in[0,1] ; \\
\lim _{r \rightarrow 0+} \frac{f(r)}{r}=\beta>0 ; \\
f(0)=f(1)=0 ; \quad 0<f(r) \leq \beta r, \quad r \in(0,1) .
\end{gathered}
$$

Then

$$
F 1=F 0=0 \leq F v \leq \beta v, \quad 0 \leq v \leq 1 .
$$

We will also assume that, for some $\rho>0$,

$$
a(x) \geq(1-\alpha) k \beta a^{-}(x)+\rho \mathbb{1}_{B_{\rho}(0)}(x), \quad x \in \mathbb{R}^{d} ;
$$

then the comparison principle holds, [32, Examples 1-3].

In Subsection 2.1 below, we consider assumptions on general reactions $F$ : $E \rightarrow E$ we can cover. In particular, we need that

$$
\frac{F v}{v} \rightarrow \beta>0 \quad \text { as } \quad v \rightarrow 0+
$$

(both convergences are in $E$ ), that holds for the reaction given by (1.4)-(1.5). Because of $F 0=0$, we get then that the Fréchet derivative of $F$ must be a (strictly positive) constant mapping. Hence, we do not allow the degenerate reaction $F^{\prime}(0)=0$, see e.g. [2].

\subsection{Description of results}

We will say that sets $\left\{\Lambda(t) \subset \mathbb{R}^{d}, t>0\right\}$ describe the propagation of the front for a solution $u=u(x, t)$ to (1.1) if, for all (small enough) $\varepsilon>0$, the following convergences hold:

$$
\begin{aligned}
& \lim _{t \rightarrow \infty} \underset{x \in \Lambda(t-\varepsilon t)}{\operatorname{essinf}} u(x, t)=1, \\
& \lim _{t \rightarrow \infty} \operatorname{ess\operatorname {sup}} u(x, t)=0 .
\end{aligned}
$$


Informally speaking, for large times, the solution $u$ becomes arbitrary close to 1 inside the set $\Lambda(t)$ and $u$ becomes arbitrary close to 0 out of this set. The intermediate zone $\Lambda(t+\varepsilon t) \backslash \Lambda(t-\varepsilon t)$ in (1.9)-(1.10) is said to be the front, or the transition zone. It can also expand as $t \rightarrow \infty$; moreover, in the accelerated case considered in the present article, it will be even with necessity, see [39].

The propagation (1.9)-(1.10) of the front, is said to have a constant speed (or just is linear in time), if $\Lambda(t)=t \Lambda(1)$. Here and below $t B:=\{t x: x \in B\}$ for a $B \subset \mathbb{R}^{d}$. In contrast, the effect of an infinite speed of propagation, see $[37,39,59]$, is called sometimes in literature an acceleration of the propagation, having in mind, for example, that then $\Lambda(t)=\eta(t) \Lambda(1)$ with $\frac{\eta(t)}{t} \rightarrow \infty, t \rightarrow \infty$.

In the present paper the propagation will be described by the sets

$$
\Lambda(t)=\Lambda(t, c):=\left\{x \in \mathbb{R}^{d} \mid c(x) \geq e^{-\beta t}\right\}, \quad t>0
$$

where the function $c: \mathbb{R}^{d} \rightarrow(0, \infty)$ will be appropriately chosen below.

We start the explanation from a more demonstrative radially symmetric case. Namely, let the kernel be a radially symmetric function:

$$
a(x)=b(|x|), \quad x \in \mathbb{R}^{d} ;
$$

and let the initial condition take either of forms:

$$
u_{0}(x)=q(|x|), \quad x \in \mathbb{R}^{d}
$$

or

$$
u_{0}(x)=\int_{\Delta(x)} q(|y|) d y, \quad x \in \mathbb{R}^{d} .
$$

Here and in the sequel, $|x|$ denotes the Euclidean norm of an $x \in \mathbb{R}^{d}$, and

$$
\Delta(x):=\left\{y \in \mathbb{R}^{d}: y_{j} \geq x_{j}, 1 \leq j \leq d\right\}, \quad x \in \mathbb{R}^{d} .
$$

We suppose also that either of functions $b, q: \mathbb{R}_{+} \rightarrow \mathbb{R}_{+}$(or both) in (1.12)(1.14) have regular heavy tails at $\infty$. Namely, we describe in Definition 2.7 below a class $\mathcal{E}_{d}$ of functions $p: \mathbb{R}_{+} \rightarrow \mathbb{R}_{+}$such that, in particular,

$$
\lim _{s \rightarrow \infty} e^{k s} p(s)=\infty, \quad k>0,
$$

i.e. each $p \in \mathcal{E}_{d}$ decays slowly than any exponential function. The class $\mathcal{E}_{d}$ contains any function which is decreasing at $\infty$ to 0 and is asymptotically proportional at $\infty$ to either of

$$
\begin{array}{ll}
(\log s)^{\lambda} s^{-(d+\delta)}, & s^{\nu}(\log s)^{\mu} \exp \left(-c(\log s)^{1+\delta}\right), \\
s^{\nu}(\log s)^{\mu} \exp \left(-c s^{\gamma}\right), & s^{\nu}(\log s)^{\mu} \exp \left(-c \frac{s}{(\log s)^{1+\delta}}\right),
\end{array}
$$

provided that $\delta, c>0, \gamma \in(0,1), \mu, \nu \in \mathbb{R}$, and $\lambda \in \mathbb{R}$ for $d=1$, however, $\lambda=0$ for $d>1$.

Theorem 1.1. Let $\beta>0$ be fixed and $F: E \rightarrow E$ be given by (1.4). Suppose that (1.5), (1.7) hold. Let $b, q: \mathbb{R}_{+} \rightarrow \mathbb{R}_{+}$be such that, for some $M, r, \delta, \rho>0$,

$$
\begin{aligned}
& \qquad b(s)+q(s) \leq \frac{M}{(1+s)^{d+1+\delta}}, \quad s \geq r ; \quad q(s) \geq \rho, \quad s \in[0, \rho] . \\
& \text { Let } a(x)=b(|x|), x \in \mathbb{R}^{d} \text {. Define } c: \mathbb{R}^{d} \rightarrow \mathbb{R}_{+} \text {as follows. }
\end{aligned}
$$


1. If $u_{0}(x)=q(|x|) \in[0,1], x \in \mathbb{R}^{d}$, we assume that, for some $\gamma>0$, either

(i) $\gamma u_{0}(x) \leq a(x)=: c(x), x \in \mathbb{R}^{d}$, and $b \in \mathcal{E}_{d}$; or,

(ii) $\gamma a(x) \leq u_{0}(x)=: c(x), x \in \mathbb{R}^{d}$, and $q \in \mathcal{E}_{d}$.

2. If $u_{0}(x)=\int_{\Delta(x)} q(|y|) d y, x \in \mathbb{R}^{d}$, with $\int_{0}^{\infty} q(s) s^{d-1} d s \in(0,1]$, we assume that, for some $\gamma>0$, either

(i) $\gamma u_{0}(x) \leq \int_{\Delta(x)} a(y) d y=: c(x), x \in \mathbb{R}^{d}$, and $b \in \mathcal{E}_{d}$; or,

(ii) $\gamma \int_{\Delta(x)} a(y) d y \leq u_{0}(x)=: c(x), x \in \mathbb{R}^{d}$, and $q \in \mathcal{E}_{d}$.

Then (1.9)-(1.10) hold with $\Lambda(t)=\Lambda(t, c)$ given by (1.11).

Informally speaking, $c$ in (1.9)-(1.11) is

either $a$ or $u_{0}$, whichever decays slower, if (1.13) holds;

either $\int_{\Delta(x)} a$ or $u_{0}$, whichever decays slower, if (1.14) holds.

On the sketches below, we shade the sets $\Lambda(t-\varepsilon t)$ and $\mathbb{R}^{d} \backslash \Lambda(t+\varepsilon t)$ for both types of initial conditions (1.13)-(1.14).

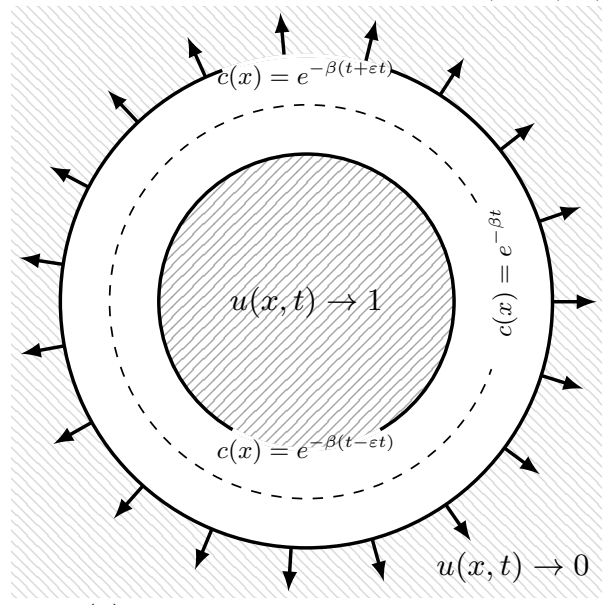

(a) Boundaries for level sets for $u_{0}$ given by (1.13)

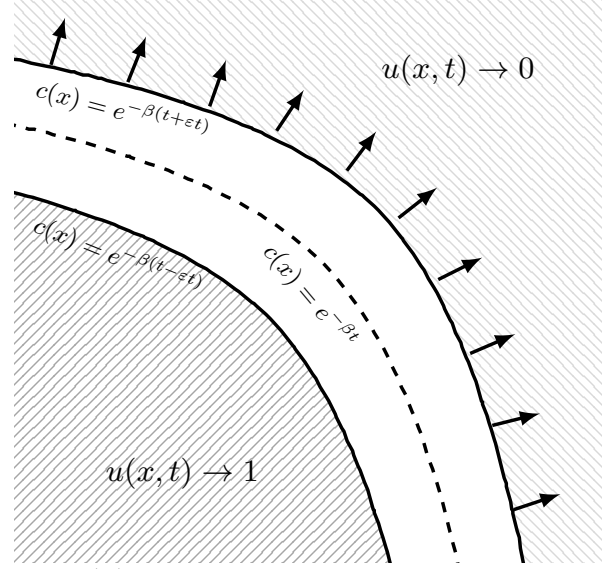

(b) Boundaries for level sets for $u_{0}$ given by (1.14)

Figure 1: Boundaries for level sets $\Lambda(t), \Lambda(t-\varepsilon t), \Lambda(t+\varepsilon t)$ for two classes of initial conditions $u_{0}$ (in $\mathbb{R}^{2}$ ); the arrows show the directions of the propagation

In Section 2 below, we generalize Theorem 1.1 by considering, instead of (1.4), a class of reactions in (1.1) which satisfy (1.6), (1.8). Moreover, we weaken the assumption that the kernel and the initial condition in (1.12)-(1.14) are constructed by radially symmetric functions, by allowing that each of $a=a(x)$ and $u_{0}=u_{0}(x)$ may fluctuate in an appropriate way. To demonstrate possible fluctuations, we present the examples below.

Example 1.2. We start with the case when $u_{0} \in L^{1}\left(\mathbb{R}^{d}\right)$, cf. (1.13). Then $c$ in (1.9)-(1.11) will be chosen in the form $c(x)=b(|x|), x \in \mathbb{R}^{d}$, with a decreasing at $\infty$ function $b: \mathbb{R}_{+} \rightarrow \mathbb{R}_{+}$. In this case, the set (1.11) is given by

$$
\Lambda(t, c)=\left\{x \in \mathbb{R}^{d}|| x \mid \leq \eta(t)\right\}, \quad \eta(t):=b^{-1}\left(e^{-\beta t}\right)
$$


for large enough $t$ (to invert $b$ ). We assume that $b_{\max }, b_{\min }: \mathbb{R}_{+} \rightarrow \mathbb{R}_{+}$and $r>0$ are such that the following two conditions hold:

$$
\max \left\{a(x), u_{0}(x)\right\} \leq b_{\max }(|x|), \quad|x| \geq r ;
$$

either $a(x) \geq b_{\min }(|x|), \quad|x| \geq r, \quad$ or $u_{0}(x) \geq b_{\min }(|x|), \quad|x| \geq r$.

Then (1.9)-(1.10) hold with $\Lambda(t)=\Lambda(t, c)$ given by (1.2), where $\eta(t)$ can be found from the following table:

\begin{tabular}{|c|c|c|}
\hline$b_{\min }(|x|)$ & $b_{\max }(|x|)$ & $\eta(t)$ \\
\hline$\frac{1}{(\log |x|)^{\nu}} \frac{1}{|x|^{d+\mu}}$ & $(\log |x|)^{\nu} \frac{1}{|x|^{d+\mu}}$ & $\exp \left(\frac{\beta t}{d+\mu}\right)$ \\
\hline$\frac{1}{|x|^{\nu}} \exp \left(-(\log |x|)^{\lambda}\right)$ & $|x|^{\nu} \exp \left(-(\log |x|)^{\lambda}\right)$ & $\exp \left((\beta t)^{\frac{1}{\lambda}}\right)$ \\
\hline$\frac{1}{|x|^{\nu}} \exp \left(-|x|^{\gamma}\right)$ & $|x|^{\nu} \exp \left(-|x|^{\gamma}\right)$ & $(\beta t)^{\frac{1}{\gamma}}$ \\
\hline$\frac{1}{|x|^{\nu}} \exp \left(-\frac{|x|}{(\log |x|)^{\lambda}}\right)$ & $|x|^{\nu} \exp \left(-\frac{|x|}{(\log |x|)^{\lambda}}\right)$ & $\sim \beta t(\log t)^{\lambda}$ \\
& & $t \rightarrow \infty$ \\
\hline
\end{tabular}

Here $\nu \geq 0, \mu>0, \lambda>1, \gamma \in(0,1)$. For the first three cases, the calculation of $\eta(t)$ is straightforward; in the last case, the asymptotic of $\eta(t)$ is shown in the Appendix, Lemma A.1.

In the following two examples, we consider the case of a non-integrable $u_{0}$, cf. (1.14).

Example 1.3. Let $d=1$. Then (1.9)-(1.11) hold with

$$
\Lambda(t)=(-\infty, \eta(t)], \quad \eta(t):=c^{-1}\left(e^{-\beta t}\right), \quad c(x)=\int_{x}^{\infty} b(y) d y
$$

for large $t$ and $x$, and for a decreasing at $\infty$ function $b \in L^{1}\left(\mathbb{R}_{+} \rightarrow \mathbb{R}_{+}\right)$.

For example, let, for some $\mu>0$ and $\nu \geq 0$,

$$
\frac{1}{(\log |x|)^{\nu}} \frac{1}{|x|^{1+\mu}} \leq a(x) \leq(\log |x|)^{\nu} \frac{1}{|x|^{1+\mu}}
$$

for large $|x|$; and suppose, for simplicity, that $u_{0}$ is monotone on $\mathbb{R}$ and, for some $\zeta \in(0,1)$,

$$
\zeta \mathbb{1}_{\mathbb{R}_{-}}(x) \leq u_{0}(x) \leq \zeta \int_{x}^{\infty} a(y) d y \leq 1, \quad x \in \mathbb{R} .
$$

Then one can choose $b(s)=s^{-1-\mu}($ for large $s)$, and then, for large $t$,

$$
\eta(t)=\exp \left(\frac{\beta t}{\mu}\right)
$$

i.e. the front propagates a bit faster than in the case of $u_{0} \in L^{1}(\mathbb{R})$. 
Example 1.4. Let $d=2$. Then the boundary of $\Lambda(t)$ is described, cf. Figure 1, for each direction $\left(\xi_{1}, \xi_{2}\right) \in S^{1}$ (the unit circle in $\mathbb{R}^{2}$ ), by two functions, $X_{1}(t)=$ $X_{1}\left(t, \xi_{1}, \xi_{2}\right)$ and $X_{2}(t)=X_{2}\left(t, \xi_{1}, \xi_{2}\right)$, such that

$$
\int_{X_{1}(t)}^{\infty} \int_{X_{2}(t)}^{\infty} b(|y|) d y_{1} d y_{2}=e^{-\beta t}, \quad|y|=\sqrt{y_{1}^{2}+y_{2}^{2}},
$$

for a decreasing at $\infty$ function $b: \mathbb{R}_{+} \rightarrow \mathbb{R}_{+}$, such that $\int_{\mathbb{R}_{+}} b(r) r d r<\infty$. Consider the acceleration of the front propagation along the diagonal direction $\xi_{1}=\xi_{2}=\frac{1}{\sqrt{2}}$; we set then $X(t):=X_{1}(t)=X_{2}(t)$.

Let, for example, for $\nu \geq 0$,

$$
\frac{1}{|x|^{\nu}} \exp (-\sqrt{|x|}) \leq a(x) \leq|x|^{\nu} \exp (-\sqrt{|x|})
$$

for large $|x|$; and suppose that, for some $\zeta \in(0,1)$ and a.a. $\left(x_{1}, x_{2}\right) \in \mathbb{R}^{2}$,

$$
\zeta \mathbb{1}_{\mathbb{R}_{-}^{2}}\left(x_{1}, x_{2}\right) \leq u_{0}\left(x_{1}, x_{2}\right) \leq \zeta \int_{x_{1}}^{\infty} \int_{x_{2}}^{\infty} a\left(y_{1}, y_{2}\right) d y_{1} d y_{2} \leq 1 .
$$

Then, for each $\varepsilon \in(0,1)$ and for all large enough $t$,

$$
\frac{1}{2 \sqrt{2}} \beta^{2}(1-\varepsilon)^{2} t^{2} \leq X(t) \leq \frac{1}{\sqrt{2}} \beta^{2} t^{2}
$$

see Lemma A.2 and Remark A.3 in the Appendix for details.

\subsection{Overview of literature}

In the recent decades, there is a growing interest to the study of nonlocal monostable reaction-diffusion equations: for a pure local reaction (1.4) with $\alpha=1$, see e.g. $[1,5,9,16,17,37,54,55,58,59]$; for a pure nonlocal reaction (1.4) with $\alpha=0$ and $k=1$, see $[20,27-30,36,52]$; and for the origins of the topic, see also $[4,19,49,50,53,57]$.

Two classical examples which satisfy $(1.5)$ are $f(s)=\nu s(1-s)$, cf. [34], and $f(s)=\nu s(1-s)^{2}$, cf. [43]; for some $\nu>0$. Note that, if $f$ in (1.5) is differentiable at 0 , then we require $f^{\prime}(0)=\beta>0$ and $f(u) \leq f^{\prime}(0) u$ for all $0 \leq u \leq 1$. The importance of the latter assumption for the front propagation was pointed out in e.g. $[9,17]$, it leads to the possibility to describe the front using the linearized version of the corresponding equation (1.1) about 0 , that is just (3.1) below; note also that this assumption can be weaken, see [56]. The degenerate case $f^{\prime}(0)=0$ was considered in e.g. $[2,60]$

Now we discuss the existing results for both linear in time and accelerated propagations.

Mollison $[49,50]$ studied, for the dimension $d=1$, a local reaction $F$ given by (1.4) with $\alpha=0, k=1, a^{-}=a$, and $\beta=1$, for a monotone initial condition $u_{0}$, cf. (1.14). He has shown that the property of the corresponding propagation front to have an 'averaged' constant speed is necessary and sufficient with the existence of a $\lambda>0$, such that

$$
\int_{\mathbb{R}} a(s) e^{\lambda s} d s<\infty, \quad \sup _{s \in \mathbb{R}} u_{0}(s) e^{\lambda s}<\infty .
$$


Note that such $u_{0}$ gives an unbounded set $\Lambda(t)=(-\infty, \gamma t]$ in (1.9)-(1.10).

For $d \geq 1$, we have shown in [30, Proposition 3.1] that similar restrictions

$$
\exists \mu>0: \int_{\mathbb{R}^{d}} a(x) e^{\mu|x|} d x<\infty, \quad \forall \lambda>0: \operatorname{ess~sup}_{x \in \mathbb{R}^{d}} u_{0}(x) e^{\lambda|x|}<\infty
$$

yield that the solution $u$ to the equation (1.1) propagates at most linearly in any direction $\xi \in \mathbb{R}^{d}$. Note that (1.20) implies $u_{0} \in L^{1}\left(\mathbb{R}^{d}\right)$, cf. (1.13). Moreover, for the reaction $F$ given by (1.4) with $k=1$ and under (1.7), we have proved in [30, Propositions 4.7 and 4.2] that the assumption (1.20) implies that the convergences (1.9)-(1.10) hold with $\Lambda(t)=t \Lambda(1)$, where $\Lambda(1)$ is a bounded convex subset of $\mathbb{R}^{d}$. For the particular case of $\alpha=0, k=1, a=a^{-}$in (1.4), a similar result was obtained in [52].

The conditions (1.20) are closed to the necessary ones, cf. [37,59]. We have proved in [30, Proposition 1.4] (cf. also [37]) that, if a bit weaker form of (1.20) fails for $a$ (roughly, if $a$ is 'heavier' than any exponent at infinity), then the convergence (1.9) holds with $\Lambda(t)=t K$ for an arbitrary compact set $K \subset \mathbb{R}^{d}$. Therefore, the propagation of the front is faster than linear.

For the dimension $d=1$ and for the local reaction $F$ given by (1.4) with $\alpha=1$, the acceleration was known in mathematical biology, see e.g. the results and references in $[38,45,47]$. The first rigorous result in this direction was done by Garnier [37], who proved an analogue of (1.9)-(1.10) for $d=1$ and a compactly supported initial condition $u_{0}$. However, in his approach, the set $\Lambda(t+\varepsilon t, c)$ in (1.10) given by (1.11) was replaced by $\Lambda(\gamma t, c)$ with some (unknown) $\gamma>1$, i.e. the result was not sharp. Note that the technique in [37] was inspired by [40], where an acceleration was shown for the classical KPP-equation with a slowly decreasing initial condition; see also a recent paper [41].

Further progress in the study of the acceleration in the dimension $d=1$, for a local reaction $F$ (with $\alpha=1$ in (1.4)), was done recently. In [10], both $a$ and $u_{0}$ are supposed to be symmetric, with a heavy-tailed $a$; the technique used there goes back to [21]. In [2], the case of $f^{\prime}(0)=0$ was considered (that is not covered by the present paper, because of (1.8)), then $a$ does not need to be symmetric, and $u_{0}$ is separated from 0 at $-\infty$, however, $u_{0}(x)=0$ for large $x$.

In [31], we considered, for the case $d=1$, a general reaction $F$ which satisfies (1.6)-(1.8) and fulfills the assumptions of Section 2 below. In this case, the result of Theorem 1.1 was extended to functions $a$ and $u_{0}$ which have different orders of decreasing at $\pm \infty$; for example, $a(x)=\exp \left(-x^{\gamma}\right), x>r$, and $a(x)=(1-x)^{-1-\delta}$, $x<-r$, for some $\gamma \in(0,1), \delta, r>0$.

Therefore, up to our knowledge, the present paper is the first one which contains results about the acceleration in (1.9)-(1.10) for the multidimensional case $d>1$.

Note also that analogous results were obtained in $[14,15]$ for the equation of the type (1.1) with a local reaction $F$, where $L$ in (1.2), was replaced by a fractional Laplacian (in particular, the kernel $a$ had a non-integrable singularity at the origin); cf. also $[13,24,48]$. For the acceleration in integrodifference equations see also [46].

The approach we follow in the paper is similar to the one we applied to the one-dimentional case $(d=1)$ in [30]. However, the generalization to $d>1$ is not trivial at all. The core of our techniques consists of estimates on the convolution $a * u$ in terms of the asymptotic properties of $a$ and $u$ at infinity. In the one- 
dimensional case, the idea was to choose an appropriate positive funciton $h$, which allows to estimate each of the following summands separately,

$$
(a * u)(x)=\left(\int_{-\infty}^{-h(x)}+\int_{-h(x)}^{h(x)}+\int_{h(x)}^{\infty}\right) a(x-y) b(y) d y .
$$

For $d>1$, natural analogues of $(-\infty,-h],[-h, h],[h, \infty)$ are $\times_{j=1}^{d}(-\infty,-h]$, $\mathbf{X}_{j=1}^{d}[-h, h], \mathbf{X}_{j=1}^{d}[h, \infty)$, respectively. However, these sets do not cover $\mathbb{R}^{d}$ for any $d>1$. For $d>1$, an analogue of (1.21) involves additional terms, analysis of which is non-trivial and can not be reduced to the one-dimensional case, even for radially symmetric functions. Note also that for radially symmetric $a(x)=b_{1}(|x|)$ and $u(x)=b_{2}(|x|)$, one gets $(a * u)(x)=b_{3}(|x|)$, however, up to our knowledge, there are not any results (for $d>1$ ) about the asymptotics of $b_{3}$ in terms of $b_{1}$ and $b_{2}$. As a result, extension of the results to $d>1$ appeared far from being tedious.

We would like to mention also that assumption (1.7) (essential for the case of nonlocal reaction) implies that the comparison principle holds. If, however, comparison fails then solution's behaviour becomes more subtle. In particular, the bifurcation of the constant solution $u \equiv 1$ is possible, developing an infinite family of spatially periodic stationary solutions; see [18] and also [7, 12, 22, 23].

The present paper is organized as follows. In Subsection 2.1, we formulate further assumptions on $a$ and $F$ and known results about solutions to (1.1). Note that these assumptions are fulfilled for the reaction (1.4) in conditions of Theorem 1.1, see Lemma 2.5. In Subsection 2.2, we describe the mentioned class $\mathcal{E}_{d}$ of regular heavy-tailed functions and formulate the main result, Theorem 2.9, which generalizes Theorem 1.1 (cf. Remark 2.10). In Section 3, we present a scheme of the proof for Theorem 2.9. Section 4 contains technical tools, mainly about the properties of sets $\Lambda(t)$ in (1.9)-(1.11). In Section 5, we present a detailed proof of Theorem 2.9. Finally, the Appendix contains, in particular, the proof of the mentioned Lemma 2.5.

\section{Assumptions and general results}

\subsection{General assumptions}

Let $\beta>0$ be fixed and consider $F: E \rightarrow E$ which satisfies (1.6), (1.8). Then the mapping $G v:=\beta v-\frac{F v}{v}, v \in E$, is well-defined at least for $0<v \leq 1$. It will be more convenient for us to formulate assumptions below in terms of the mapping $G$ rather than $F$.

Namely, we start now with the equation

$$
\left\{\begin{array}{l}
\frac{\partial}{\partial t} u(x, t)=\int_{\mathbb{R}^{d}} a(x-y) u(y, t) d y-m u(x, t)-u(x, t)(G u)(x, t), \\
u(x, 0)=u_{0}(x) .
\end{array}\right.
$$

Here $m>0$ is a constant, and $G$ is a nonnegative continuous mapping on $E$ which is acting in $x$, i.e. $(G u)(x, t):=(G u(\cdot, t))(x) \geq 0$ for $u \geq 0$.

We will assume that:

$$
\beta:=1-m>0
$$




$$
\begin{gathered}
\text { for each } 0 \leq v \leq 1, \\
0=G 0 \leq G v \leq G 1=\beta .
\end{gathered}
$$

Then, by setting

$$
F v:=v(\beta-G v), \quad v \in E,
$$

we get (1.6), (1.8). Therefore, we can rewrite the equation (2.1) in the form (1.1).

Note also that, actually, in the right hand side of (2.1) we separate (in the first two terms) the linearization of the reaction $F$ at 0 .

The solution $u(x, t)$ to $(2.1)$ may be interpreted as the local density of an evolving in time system of entities which reproduce themselves, compete, and die. The reproduction appears according to the dispersion, which is realized via the fecundity rate 1 and the density $a$ of a probability dispersion distribution. The death may appear due the constant inner mortality $m>0$ within the system, as well as due to the density dependent rate $G u$, which describes a competition within the system. For the derivation of the equation (2.1) from the underlying population dynamics (in the case $G u=\beta a^{-} * u$ ) see e.g. $[8,26,36]$ and references therein.

Consider further assumptions to (A1)-(A2). Set, for an $r>0$,

$$
E^{+}:=\{v \in E \mid v \geq 0\}, \quad E_{r}^{+}:=\{v \in E \mid 0 \leq v \leq r\} .
$$

We assume that $G$ is (locally) Lipschitz continuous in $E_{1}^{+}$, namely,

$$
\begin{gathered}
\text { there exists } l>0 \text { such that } \\
\|G v-G w\| \leq l\|v-w\|, \quad v, w \in E_{1}^{+} .
\end{gathered}
$$

We restrict ourselves to the case when the comparison principle for $(2.1)$ holds. Namely, we assume that the right hand side (r.h.s. in the sequel) of (2.1) is a (quasi-) monotone operator:

$$
\begin{gathered}
\text { for some } p \geq 0 \text { and for any } v, w \in E_{1}^{+} \text {with } v \leq w, \\
a * v-v G v+p v \leq a * w-w G w+p w .
\end{gathered}
$$

Here and below $*$ means the classical convolution over $\mathbb{R}^{d}$, i.e., for $u \in E$,

$$
(a * u)(x):=\int_{\mathbb{R}^{d}} a(x-y) u(y) d x, \quad x \in \mathbb{R}^{d} .
$$

Theorem 2.1 ([32, Theorems 2.2, 2.3, Proposition 4.2]). Let (A1)-(A4) hold. Let $u_{0} \in E_{1}^{+}$. Then, for each $T>0$, there exists a unique solution $u=u(x, t)$ to (2.1) for $t \in[0, T]$; and (1.3) holds for all $t>0$. Moreover, if $v_{0} \in E_{1}^{+}$, $v=v(x, t)$ is the corresponding solution to (2.1), and if $u_{0} \leq v_{0}$, then $0 \leq$ $u(\cdot, t) \leq v(\cdot, t) \leq 1$ for all $t>0$.

Note also that if $u_{0}$ is (uniformly) continuous function on $\mathbb{R}^{d}$, then $u(\cdot, t)$ will be also (uniformly) continuous function on $\mathbb{R}^{d}$ for all $t>0$. The comparison between solutions in Theorem 2.1 was a part of a more general result, which we will also use. 
Theorem 2.2 ([32, Theorems 2.2]). Let (A1)-(A4) hold. Let $T>0$ be fixed. Suppose that $u_{1}, u_{2}:[0, T] \rightarrow E$ are continuous mappings, continuously differentiable in $t \in(0, T]$, and such that, for $(x, t) \in \mathbb{R}^{d} \times(0, T]$,

$$
\begin{gathered}
\frac{\partial u_{1}}{\partial t}-a * u_{1}+m u_{1}+u_{1} G u_{1} \leq \frac{\partial u_{2}}{\partial t}-a * u_{2}+m u_{2}+u_{2} G u_{2}, \\
u_{1}(x, t) \geq 0, \quad u_{2}(x, t) \leq 1, \quad 0 \leq u_{1}(x, 0) \leq u_{2}(x, 0) \leq 1 .
\end{gathered}
$$

Then $u_{1}(x, t) \leq u_{2}(x, t)$ for $(x, t) \in \mathbb{R}^{d} \times[0, T]$.

Note that here and in the sequel, with an abuse of notations, the symbol * stands for the convolution in $x$ variable only, when $u=u(x, t)$ in (2.3).

We assume also that the kernel $a$ is not degenerate at the origin, namely,

$$
\text { there exists } \rho>0 \text { such that } a(x) \geq \rho \text { for a.a. } x \in B_{\rho}(0) \text {. }
$$

Consider on $E$ the topology of the locally uniform convergence: a sequence $\left(v_{n}\right)_{n \in \mathbb{N}} \subset E$ is said to be convergent to $v \in E$ locally uniformly, which we denote $v_{n} \stackrel{\text { loc }}{\Longrightarrow} v, n \rightarrow \infty$, if, for each compact set $\Lambda \subset \mathbb{R}^{d}$, the sequence $\left(\mathbb{1}_{\Lambda} v_{n}\right)_{n \in \mathbb{N}}$ converges to $\mathbb{1}_{\Lambda} v$ in $E$ as $n \rightarrow \infty$; here and below $\mathbb{1}_{B}$ denotes the indicator-function of a $B \subset \mathbb{R}^{d}$.

Stability of the solution to (2.1) with respect to the initial condition in the topology of the locally uniform convergence requires continuity of $G$ in this topology. Namely, we assume that

$$
\text { for any } v_{n}, v \in E_{1}^{+} \text {such that } v_{n} \stackrel{\text { loc }}{\Longrightarrow} v, n \rightarrow \infty \text {, one has }
$$

$$
G v_{n} \stackrel{\text { loc }}{\Longrightarrow} G v, n \rightarrow \infty \text {. }
$$

We will consider the translation invariant case only. Namely, for each $y \in \mathbb{R}^{d}$, we denote by $T_{y}: E \rightarrow E$ the translation operator, that is $\left(T_{y} v\right)(x):=v(x-y)$, $x \in \mathbb{R}^{d}$. Then we assume that

$$
\left(T_{y} G v\right)(x)=\left(G T_{y} v\right)(x), \quad v \in E_{1}^{+}, x \in \mathbb{R}^{d} .
$$

Under (A7), for any $r \equiv$ const $\in(0,1), G r \equiv$ const. In this case, we assume also that

$$
G r<\beta, \quad r \in(0,1) .
$$

Finally, we will distinguish two cases. If the condition

$$
\int_{\mathbb{R}^{d}}|y| a(y) d y<\infty
$$

holds, then we assume, additionally to (A4), that

$$
\begin{gathered}
\text { there exist } p \geq 0, b \in C^{\infty}\left(\mathbb{R}^{d}\right) \cap L^{\infty}\left(\mathbb{R}^{d}\right), \delta>0 \text { such that } \\
a(x)-b(x) \geq \delta \mathbb{1}_{B_{\delta}(0)}(x), \quad x \in \mathbb{R}^{d}, \\
w G w \leq b * w+p w, \quad w \in E_{1}^{+},
\end{gathered}
$$

and also

$$
\int_{\mathbb{R}^{d}} x a(x) d x=0 \in \mathbb{R}^{d} .
$$


Otherwise, if (A9) does not hold, then we assume that,

$$
\begin{gathered}
\text { let (A1)-(A4) hold, and for each } n \in \mathbb{N} \text {, there exist } \\
0 \leq a_{n} \in L^{1}\left(\mathbb{R}^{d}\right) \text { with } \int_{\mathbb{R}^{d}} a_{n}(x) d x>m, \\
G_{n}: E \rightarrow E \text { and } \theta_{n} \in(0,1] \text { with } G_{n} \theta_{n}=\beta \\
\text { which satisfy analogues of (A2)-(A11) } \\
\text { (with the } 0 \leq u \leq 1 \text { repalced by } 0 \leq u \leq \theta_{n} \text { ) } \\
\text { such that } \theta_{n} \rightarrow 1, n \rightarrow \infty, \text { and } \\
a_{n} * w-w G_{n} w \leq a * w-w G w, \quad w \in E_{\theta_{n}}^{+}, n \in \mathbb{N} .
\end{gathered}
$$

The following statement describes the so-called hair-trigger effect.

Theorem 2.3 (cf. [32, Theorems 2.5, 2.7]). Let either (A1)-(A11) hold or (A12) hold. Let $u_{0} \in E_{1}^{+}, u_{0} \not \equiv 0$, and let $u=u(x, t)$ be the corresponding solution to (2.1). Then, for each compact set $K \subset \mathbb{R}^{d}$,

$$
\lim _{t \rightarrow \infty} \operatorname{essinf}_{x \in K} u(x, t)=1 .
$$

Remark 2.4. For a brevity of notations, we will treat $u_{0} \not \equiv 0$ as follows: there exist $\rho>0$ and $x_{0} \in \mathbb{R}^{d}$ such that $u_{0}(x) \geq \rho$ for a.a. $x \in B_{\rho}\left(x_{0}\right)$.

The following lemma shows that the mapping $G$ corresponding to the reaction (1.4) satisfies the assumptions above. Its proof is provided in the Appendix.

Lemma 2.5. Let (A1), (A9) and (A11) hold. Let F be given by (1.4). Suppose that, for some $\rho>0,(1.7)$ holds, and, if $\alpha \neq 0$, suppose also that (1.5) holds. Set, for a.a. $x \in \mathbb{R}^{d}$,

$$
(G u)(x):=\beta-\frac{(F u)(x)}{u(x)}, \quad u(x) \neq 0, \quad(G u)(x):=0, \quad u(x)=0 .
$$

Then (A2)-(A8) and (A10) hold.

\subsection{General results}

In this Subsection, we present a general result, Theorem 2.9, which generalizes Theorem 1.1 to a non radially symmetric case and to the case of a reaction $F$ in (1.1) which corresponds to a mapping $G: E \rightarrow E$ in (2.1) which fulfills the assumptions above. We start with a description of the class $\mathcal{E}_{d}$ of functions on $\mathbb{R}_{+}$which is used in the both Theorems.

Definition 2.6. Let a measurable function $b: \mathbb{R}_{+} \rightarrow \mathbb{R}_{+}$be such that $b(s)>0$ for all $s \geq \rho$ with some $\rho=\rho_{b} \geq 0$. Then the function $b$ is said to be (right-side)

- long-tailed, if, for any $\tau \geq 0$,

$$
\lim _{s \rightarrow \infty} \frac{b(s+\tau)}{b(s)}=1
$$

- tail-decreasing, if $b=b(s)$ is strictly decreasing on $[\rho, \infty)$ to 0 ; 
- tail-log-convex, if the function $\log b$ is convex (and hence continuous) on $(\rho, \infty)$.

Definition 2.7. Let $d \in \mathbb{N}$.

1) Let $\mathcal{D}_{d}$ be the set of all bounded tail-decreasing functions $b: \mathbb{R}_{+} \rightarrow(0, \infty)$ such that $\int_{0}^{\infty} b(s) s^{d-1} d s<\infty$ and $\inf _{|s|<r} b(s)>0$ for each $r>0$;

2) Let $\mathcal{E}_{d} \subset \mathcal{D}_{d}$ be the set of all functions $b \in \mathcal{D}_{d}$ such that

- $b$ is long-tailed and tail-log-convex, and $b(\rho) \leq 1$ (without loss of generality);

- there exist $\delta=\delta_{b} \in(0,1)$ and an increasing function $h=h_{b}:(0, \infty) \rightarrow$ $(0, \infty)$, with $h(s)<\frac{s}{2}$ and $\lim _{s \rightarrow \infty} h(s)=\infty$ such that

$$
\lim _{s \rightarrow \infty} \frac{b(s \pm h(s))}{b(s)}=1, \quad \lim _{s \rightarrow \infty} b(h(s)) s^{1+\delta}=0 ;
$$

- and, for the case $d>1$, we assume, additionally, that either, for some $\mu, M>0$,

$$
b(s)=\frac{M}{(1+s)^{d+\mu}}, \quad s \in \mathbb{R}_{+},
$$

or, for all $\nu \geq 1$,

$$
\lim _{s \rightarrow \infty} b(s) s^{\nu}=0 .
$$

The peculiarities of functions from $\mathcal{E}_{d}$ can be found in [33].

As it was mentioned before, we are going to weaken the radially symmetric conditions of Theorem 1.1. Namely, we assume that there exist bounded measurable functions $b^{+}, b_{+}: \mathbb{R}_{+} \rightarrow \mathbb{R}_{+}$and $v^{\circ}, v_{\circ}: \mathbb{R}^{d} \rightarrow[0,1]$ such that

$$
\begin{aligned}
b_{+}(|x|) & \leq a(x) \leq b^{+}(|x|), & & x \in \mathbb{R}^{d} ; \\
v_{\circ}(x) & \leq u_{0}(x) \leq v^{\circ}(x), & & x \in \mathbb{R}^{d} .
\end{aligned}
$$

In order to control the allowed range of values for $a$ or $u_{0}$ in (B1)-(B2), we consider also the following definition.

Definition 2.8. Let $b_{1}, b_{2}: \mathbb{R} \rightarrow \mathbb{R}_{+}$and, for some $\rho \geq 0, b_{i}(s)>0$ for all $s \in[\rho, \infty), i=1,2$. The functions $b_{1}$ and $b_{2}$ are said to be (asymptotically) log-equivalent, if

$$
\log b_{1}(s) \sim \log b_{2}(s), \quad s \rightarrow \infty .
$$

We will assume also that there exist constants $M, \delta, r>0$ and a point $x_{0} \in \mathbb{R}^{d}$ such that

$$
\begin{array}{ll}
b^{+}(s) \leq \frac{M}{(1+s)^{d+\delta}}, & s \geq r ; \\
b_{+}(s) \geq \delta, & s \in[0, \delta] ; \\
v_{\circ}(x) \geq \delta, & x \in B_{\delta}\left(x_{0}\right) .
\end{array}
$$


Recall that our objective is to show (1.9)-(1.10) with $\Lambda(t)=\Lambda(t, c)$ given by (1.11). The choice of the 'shape' for the function $c=c(x)$ will be determined by the initial condition $u_{0}: \mathbb{R}^{d} \rightarrow[0,1]$. We will distinguish two cases. Namely, if the initial condition is integrable,

$$
u_{0} \in L^{1}\left(\mathbb{R}^{d}\right),
$$

then we will choose, for a suitable $b \in \mathcal{E}_{d}$,

$$
c(x)=b(|x|), \quad x \in \mathbb{R}^{d} .
$$

On contrary, if the initial condition is separated from 0 at $\underset{j=1}{\mathbb{X}}\{-\infty\}$, i.e. if

$$
\begin{gathered}
\text { there exist } \zeta \in(0,1] \text { and } z \in \mathbb{R}^{d} \text { such that } \\
u_{0}(x) \geq \zeta \mathbb{1}_{\nabla(z)}(x), \quad x \in \mathbb{R}^{d},
\end{gathered}
$$

where

$$
\nabla(z):=\left\{y \in \mathbb{R}^{d}: y_{j} \leq z_{j}, 1 \leq j \leq d\right\}, \quad z \in \mathbb{R}^{d},
$$

then we will choose (again, for some $b \in \mathcal{E}_{d}$ )

$$
c(x)=\int_{\Delta(x)} b(|y|) d y, \quad x \in \mathbb{R}^{d} .
$$

We will refer to the examples of $c(x)$ in $(\mathrm{C} 1)$ and $(\mathrm{C} 2)$ as to integrable and 'monotone' case, respectively. Note that, in the 'monotone' case, $c$ decays to 0 along all coordinate axes in $\mathbb{R}^{d}$. It is worth noting also that we have shown in [32, Proposition 5.7], that if $u_{0}$ decays along a direction in $\mathbb{R}^{d}$, then the corresponding solution decays along this direction as well.

We are ready to formulate now the general result.

Theorem 2.9. Let either (A1)-(A11) hold or (A12) hold. Let (B1)-(B5) hold. Suppose that $u_{0} \in E_{1}^{+}, u_{0} \not \equiv 0,1-u_{0} \neq \equiv 0$ (cf. Remark 2.4); and let $u$ be the corresponding solution to $(2.1)$.

1. (Front is determined by a.) Suppose that both functions $b_{+}$and $b^{+}$in (B1) belong to $\mathcal{D}_{d}$ and are log-equivalent to a function $b \in \mathcal{E}_{d}$. Let, additionally, $b_{+}$be long-tailed and tail-log-convex.

(a) Suppose that

$$
u_{0}(x) \leq b^{+}(|x|), \quad x \in \mathbb{R}^{d} .
$$

Then (1.9)-(1.10) hold with $\Lambda(t)=\Lambda(t, c)$ given by (1.11) where $c(x)$ is given by $(\mathrm{C} 1)$.

(b) Suppose that (2.10) holds and

$$
u_{0}(x) \leq \int_{\Delta(x)} b^{+}(|y|) d y, \quad x \in \mathbb{R}^{d},
$$

where $\Delta(x)$ is given by (1.15). Then (1.9)-(1.10) hold with $\Lambda(t)=$ $\Lambda(t, c)$ given by $(1.11)$ where $c(x)$ is given by $(\mathrm{C} 2)$. 
2. (Front is determined by $u_{0}$.) Consider functions $b_{\circ}, b^{\circ} \in \mathcal{D}_{d}$ which are both log-equivalent to a function $b \in \mathcal{E}_{d}$. Let, additionally, $b_{\circ}$ be long-tailed and tail-log-convex, and assume that, cf. (B1),

$$
a(x) \leq b^{\circ}(|x|), \quad x \in \mathbb{R}^{d} .
$$

(a) Suppose that

$$
v^{\circ}(x)=b^{\circ}(|x|), \quad v_{\circ}(x)=b_{\circ}(|x|), \quad x \in \mathbb{R}^{d} .
$$

Then (1.9)-(1.10) hold with $\Lambda(t)=\Lambda(t, c)$ given by (1.11) where $c(x)$ is given by $(\mathrm{C} 1)$.

(b) Suppose that

$$
v^{\circ}(x)=\int_{\Delta(x)} b^{\circ}(|y|) d y, \quad v_{\circ}(x)=\int_{\Delta(x)} b_{\circ}(|y|) d y, \quad x \in \mathbb{R}^{d} .
$$

Then (1.9)-(1.10) hold with $\Lambda(t)=\Lambda(t, c)$ given by (1.11) where $c(x)$ is given by $(\mathrm{C} 2)$.

Theorem 2.9 will be proved in Subsection 5.3 below. Note that the items 1(a) and 2(a) of Theorem 2.9 correspond to the case (2.9), and two others items correspond to (2.10).

Remark 2.10. In view of Lemma 2.5, Theorem 2.9 implies Theorem 1.1. The only observation which might to be mentioned here is that $q(s) \geq \delta, s \in[0, \rho]$, yields, cf. (2.11),

$$
\int_{\Delta(x)} q(|y|) d y \geq \mathrm{const} \cdot \mathbb{1}_{\nabla(0)}(x), \quad x \in \mathbb{R}^{d} .
$$

\section{Scheme of the proof}

In this Section, we describe the scheme of the proof for Theorem 2.9. The detailed proof is presented in Subsection 5.3 below.

We assume that (A1)-(A4) hold. Let $u_{0} \in E_{1}^{+}$and $u$ be the corresponding nonnegative solution to (2.1) according to Theorem 2.1.

\subsection{Upper estimates}

Let $w$ be the solution to the linear problem (3.1),

$$
\left\{\begin{array}{l}
\frac{\partial}{\partial t} w(x, t)=(a * w)(x, t)-m w(x, t), \\
w(x, 0)=u_{0}(x) .
\end{array}\right.
$$

The unique classical solution to (3.1) is given by $w(x, t)=e^{-m t}\left(e^{t A} u_{0}\right)(x)$, where $A v:=a * v, v \in E$ is a bounded operator on $E$. By Theorem 2.1, $u(\cdot, t) \in E_{1}^{+}$for $t \geq 0$. Then, by (A2), $(G u)(\cdot, t) \geq 0$ for $t \geq 0$, and we have, by e.g. [42, Lemma 3.3.2], that

$$
u(x, t) \leq w(x, t), \quad x \in \mathbb{R}^{d}, t \geq 0 .
$$

Below we explain the scheme of the proof for a reinforced version of (1.10) with $u$ replaced by $w$. Namely, the following statement holds. 
Theorem 3.1. Let (A1) hold. Let $0 \leq u_{0} \in E$ and $w=w(x, t)$ be the corresponding solution to the linear equation (3.1). Let $b_{1}, b_{2} \in \mathcal{D}_{d}$ be both logequivalent to a function $b \in \mathcal{E}_{d}$. Suppose that

$$
a(x) \leq b_{1}(|x|), \quad x \in \mathbb{R}^{d} .
$$

Suppose also that either

$$
u_{0}(x) \leq b_{2}(|x|), x \in \mathbb{R}^{d},
$$

and $c: \mathbb{R}^{d} \rightarrow \mathbb{R}_{+}$is given by $(\mathrm{C} 1)$, or

$$
u_{0}(x) \leq \int_{\Delta(x)} b_{2}(|y|) d y, x \in \mathbb{R}^{d}
$$

and $c$ is given by $(\mathrm{C} 2)$. Then, for any small enough $\varepsilon>0$, there exist $C_{\varepsilon}, \tau_{\varepsilon}>0$ such that

$$
\operatorname{ess}_{x \in \mathbb{R}^{d} \backslash \Lambda(t+\varepsilon t, c)} w(x, t) \leq C_{\varepsilon} e^{-\frac{\varepsilon \beta}{4} t}, \quad t \geq \tau_{\varepsilon},
$$

where $\Lambda(t, c)$ is given by $(1.11)$.

The proof of Theorem 3.1 is presented in Subsection 5.1 and it is based on the arguments below. Firstly, we prove the following statement.

Proposition 3.2. Let (3.3) holds with a function $b_{1} \in \mathcal{D}_{d}$ which is log-equivalent to a function $b \in \mathcal{E}_{d}$. Then there exists $\alpha_{1} \in(0,1)$ such that, for all $\alpha \in\left(\alpha_{1}, 1\right)$, $b^{\alpha} \in \mathcal{E}_{d}$, and both functions

$$
c_{\alpha}(x):=b(|x|)^{\alpha}, x \in \mathbb{R}^{d} ; \quad c_{\alpha}(x):=\int_{\Delta(x)} b(|y|)^{\alpha} d y, x \in \mathbb{R}^{d},
$$

satisfy the inequality

$$
\limsup _{\lambda \rightarrow 0+} \sup _{x \in\left\{c_{\alpha}<\lambda\right\}} \frac{\left(a * c_{\alpha}\right)(x)}{c_{\alpha}(x)} \leq 1 .
$$

Here and in the sequel, for $p: \mathbb{R}^{d} \rightarrow \mathbb{R}_{+}$and $\lambda>0$, we denote

$$
\{p<\lambda\}:=\left\{x \in \mathbb{R}^{d} \mid p(x)<\lambda\right\} .
$$

In other words, Proposition 3.2 shows that for both functions $c_{\alpha}$ defined by (3.7), the value of $\frac{\left(a * c_{\alpha}\right)(x)}{c_{\alpha}(x)}$ is close to 1 for appropriately large $x$.

Then, by usage of Proposition 5.3, we obtain that, for the function

$$
c_{\alpha, \lambda}(x):=\min \left\{\lambda, c_{\alpha}(x)\right\}, \quad x \in \mathbb{R}^{d}, \lambda>0,
$$

the following analogue of (3.8) holds globally: for any $\delta>0$, there exists $\lambda>0$ such that

$$
\frac{\left(a * c_{\alpha, \lambda}\right)(x)}{c_{\alpha, \lambda}(x)} \leq 1+\delta, \quad x \in \mathbb{R}^{d} .
$$

Then, using the expansion

$$
w(x, t)=e^{-m t} \sum_{n=0}^{\infty} \frac{t^{n}}{n !}\left(a^{* n} * u_{0}\right)(x),
$$


for the solution to (3.1), where $a^{* 0} * u_{0}:=u_{0}$, and the obtained global inequality $a * c_{\alpha, \lambda} \leq(1+\delta) c_{\alpha, \lambda}$, we have that if $u_{0} \leq C c_{\alpha, \lambda}$ for some $C>0$, then

$$
w(x, t) \leq C e^{(\delta+\beta) t} c_{\alpha, \lambda}(x)
$$

for all $x, t$. Therefore, on the area where $c_{\alpha, \lambda}(x) \leq e^{-\beta(1+\varepsilon) t}$, the solution $w(x, t)$ will be exponentially small in time uniformly in space (by an appropriate choice of $\delta=\delta(\varepsilon)$, see Proposition (5.4) for details). More precisely, we conclude that the following analogue of (3.6) holds, for any small enough $\varepsilon>0$,

$$
\underset{x \in \mathbb{R}^{d} \backslash \Lambda\left(t+\varepsilon t, c_{\alpha}\right)}{\operatorname{ess} \sup } w(x, t) \leq C_{\varepsilon} e^{-\frac{\varepsilon \beta}{2} t}, \quad t \geq \tau_{\varepsilon},
$$

with some $C_{\varepsilon}, \tau_{\varepsilon}>0$. Finally, for small enough $\varepsilon>0$, for large enough $t>0$, and for some $\alpha=\alpha(\varepsilon) \in\left(\alpha_{1}, 1\right)$, we will get (Proposition 4.6) that

$$
\Lambda\left(t+\frac{\varepsilon t}{2}, c_{\alpha}\right) \subset \Lambda(t+\varepsilon t, c)
$$

where $c_{\alpha}$ is given by either of (3.7) and $c$ is given by (C1) or (C2), respectively. Combining (3.11) and (3.12), we obtain (3.6).

\subsection{Lower estimates}

Consider the reaction-diffusion form (1.1)-(1.2) of (2.1). Because of the property (1.6), $(F u)(\cdot, t) \geq 0$ for $t \geq 0$. Then, by the same arguments which implied (3.2), we obtain that $u(x, t) \geq v(x, t)$ for $x \in \mathbb{R}^{d}, t \geq 0$, where $v$ solves the following linear initial value problem, cf. (3.1),

$$
\left\{\begin{array}{l}
\frac{\partial}{\partial t} v(x, t)=(a * v)(x, t)-v(x, t), \\
v(x, 0)=u_{0}(x) .
\end{array}\right.
$$

Then, clearly, $v(x, t)=e^{-t}\left(e^{t A} u_{0}\right)(x)$ with $A v=a * v$ for $v \in E$, and since, we recall, $A$ is a bounded operator on $E$, we easily conclude that

$$
u(x, t) \geq v(x, t) \geq t e^{-t}\left(a * u_{0}\right)(x), \quad x \in \mathbb{R}^{d}, t \in \mathbb{R}_{+} .
$$

More detailed arguments can be found in the proof of the following Theorem in Subsection 5.2.

Theorem 3.3. Let either (A1)-(A11) hold or (A12) hold. Let $u_{0} \in E_{1}^{+}, u_{0} \not \equiv 0$, cf. Remark 2.4; and let $u=u(x, t)$ be the corresponding solution to (2.1). Let $b \in \mathcal{D}_{d}$ be long-tailed and tail-log-convex (in particular, let $b \in \mathcal{E}_{d}$ ), and suppose that either (2.9) holds and $c(x)$ is given by $(\mathrm{C} 1)$ or (2.10) holds and $c(x)$ is given by (C2). Suppose also that

$$
\left(a * u_{0}\right)(x) \geq c(x), \quad x \in \mathbb{R}^{d} .
$$

Then, for each $\varepsilon \in(0,1)$, the convergence (1.9) holds.

Before an explanation of the scheme for the proof of Theorem 3.3, we discuss how to use it to prove the part of Theorem 2.9 regarding the convergence (1.9). 
Let (2.9) hold, that corresponds to items 1(a) and 2(a) of Theorem 2.9. It is assumed there that one of the functions $a$ and $u_{0}$ is estimated from below by $b_{\sharp}(|x|), x \in \mathbb{R}^{d}$ (where $\sharp$ was + and $\circ$, respectively) with a long-tailed and tail-log-convex $b_{\sharp} \in \mathcal{D}_{d}$, and another function is from $L^{1}\left(\mathbb{R}^{d}\right)$. In both cases, we show that (Proposition 4.11)

$$
\left(a * u_{0}\right)(x) \geq D b_{\sharp}(|x|)=: \tilde{c}(x), \quad x \in \mathbb{R}^{d},
$$

with some $D>0$.

Let now (2.10) hold. The item 1(b) of Theorem 2.9 can be easily reduced to the case $z=0 \in \mathbb{R}^{d}$ in (2.13). Then, by (B1), we obtain that, cf. (2.11),

$$
\begin{aligned}
\left(a * u_{0}\right)(x) & \geq \zeta \int_{\mathbb{R}^{d}} b_{+}(|y|) \mathbb{1}_{\nabla(0)}(x-y) d y \\
& =\zeta \int_{\Delta(x)} b_{+}(|y|) d y=: \tilde{c}(x), \quad x \in \mathbb{R}^{d} .
\end{aligned}
$$

Finally, in item 2(b) of Theorem 2.9, by the first inequality in (B2), we have, denoting $p_{\circ}(x):=b_{\circ}(|x|), x \in \mathbb{R}^{d}$, that

$$
\begin{aligned}
\left(a * u_{0}\right)(x) & \geq \int_{\mathbb{R}^{d}} a(x-y) \int_{\Delta(y)} p_{\circ}(z) d z d y \\
& =\int_{\Delta(x)}\left(a * p_{\circ}\right)(z) d z \geq D \int_{\Delta(x)} b_{\circ}(|z|) d z=: \tilde{c}(x), \quad x \in \mathbb{R}^{d},
\end{aligned}
$$

with some $D>0$, where we used again Proposition 4.11 (recall that here $b_{\circ} \in \mathcal{D}_{d}$ is supposed to be long-tailed and tail-log-convex).

Then, for either of functions $\tilde{c}(x)$ given by (3.14)-(3.16), one can apply Theorem 3.3 to get $(1.9)$ with $c$ replaced by $\tilde{c}$ and $\varepsilon$ replaced by $\frac{\varepsilon}{2}$. Finally, we show that (Proposition 4.7), if $b_{+}$or $b_{\circ}$ above is log-equivalent to a function $b \in \mathcal{D}_{d}$, then, for small enough $\varepsilon>0$ and for large enough $t>0$,

$$
\Lambda(t-\varepsilon t, c) \subset \Lambda\left(t-\frac{\varepsilon}{2} t, \tilde{c}\right)
$$

cf. (3.12), where $c$ is given by either (C1) or (C2) for the cases (2.9) and (2.10), respectively; and therefore, one gets (1.9).

And now we are going to outline the scheme of the proof for Theorem 3.3. Take any $0<\delta<\beta=1-m$. Suppose that $\varpi: \mathbb{R}^{d} \times \mathbb{R}_{+} \rightarrow \mathbb{R}_{+}$is a bounded function such that the function $v(x, t)=\lambda \varpi(x, t)$ is a sub-solution to the equation

$$
\frac{\partial}{\partial t} w(x, t)=(a * w)(x, t)-(m+\delta) w(x, t)
$$

for all small enough $\lambda>0$, cf. (3.1) and Definition 5.13 below. Next, the assumption (A3) implies the continuity of $G$ at 0 on $\{0 \leq v \in E\}$. Therefore, for any small enough $\lambda>0$, we obtain that $(G v)(\cdot, t) \leq \delta$ for $t \geq 0$. As a result,

$$
\frac{\partial}{\partial t} v-a * v+m v+v G v \leq \frac{\partial}{\partial t} v-a * v+(m+\delta) v \leq 0
$$

for large $t$, i.e. $v$ (with small $\lambda>0$ ) will be a sub-solution to (2.1) as well. See Proposition 5.17 for further details. 
Next, we show (Proposition 5.14) that the function

where

$$
v(x, t)=\frac{1}{\sigma} \int_{t}^{t+\sigma} g(x, s) d s,
$$

$$
g(x, t)=\lambda \min \left\{1, c(x) e^{\beta(1-\varepsilon) t}\right\}
$$

cf. (3.10), will be a sub-solution to (3.17) for small $\lambda, \varepsilon, \sigma>0$, provided that $c(x)$ is given by either of $(\mathrm{C} 1)$ or $(\mathrm{C} 2)$ with a long-tailed and tail-log-convex function $b \in \mathcal{D}_{d}$. Then, by the above, such $v$ is a sub-solution to (2.1). From this, by the comparison Theorem 2.2, we conclude that

$$
u(x, t) \geq \lambda, \quad x \in \Lambda(t-\varepsilon t, c)
$$

for large $t>0$ and small $\lambda, \varepsilon>0$. Finally, we cover $\Lambda(t-\varepsilon t, c)$ by compacts and apply the hair-trigger Theorem 2.3 on each of them. For the (quite technical) details, see the proof of Theorem 3.3 in Subsection 5.2 below.

\section{Technical tools}

Through this Section, $\beta>0$ is a fixed constant.

\subsection{Functions constructed by tail-decreasing functions}

Definition 4.1. If for some $b \in \mathcal{D}_{d}$ (c.f. Definition 2.7), a function $c=c(x)$ is given by either of $(\mathrm{C} 1)$ and $(\mathrm{C} 2)$, we will say that the function $c$ is constructed by the function $b$.

For any $x=\left(x_{1}, \ldots, x_{d}\right) \in \mathbb{R}^{d}$, we set

$$
\widehat{x}:=\max _{1 \leq j \leq d} x_{j} \in \mathbb{R},
$$

Remark 4.2. If $c=c(x)$ is constructed by $b \in \mathcal{D}_{d}$, then evidently the set $\Lambda(t, c)$ given by (1.11) is nonempty for big enough $t$, and the following limit holds,

$$
\lim _{t \rightarrow \infty} \sup _{x \in \mathbb{R}^{d} \backslash \Lambda(t, c)} c(x)=\lim _{t \rightarrow \infty} \sup _{x \in\left\{c<e^{-\beta t}\right\}} c(x)=0,
$$

cf. (3.9). For $c$ given by (C1), we have that $\lim _{|x| \rightarrow \infty} c(x)=0$. For $c$ given by (C2), we have, cf. (4.1),

$$
\lim _{\widehat{x} \rightarrow \infty} c(x)=\lim _{\widehat{x} \rightarrow \infty} \int_{\Delta(x)} b(|y|) d y=0 .
$$

The proof of the following Proposition is straightforward, cf. Definition 2.7 and (1.11).

Proposition 4.3. Let $c^{(i)}=c^{(i)}(x)$ be constructed by $b_{i} \in \mathcal{D}_{d}, i=1,2$ (both given simultaneously either by $(\mathrm{C} 1)$ or by $(\mathrm{C} 2))$. Suppose that there exists $\rho>0$ such that $b_{1}(s) \leq b_{2}(s)$ for all $s \geq \rho$. Then, there exists $\tau=\tau\left(b_{1}, b_{2}\right)>0$ such that $\Lambda\left(t, c^{(1)}\right) \subset \Lambda\left(t, c^{(2)}\right)$ for all $t \geq \tau$. In particular, if $b_{1}(s)=b_{2}(s)$ for all $s \geq \rho$, then $\Lambda\left(t, c^{(1)}\right)=\Lambda\left(t, c^{(2)}\right)$ for all $t \geq \tau$. 
The following proposition implies that if $c$ is constructed by $b$, then in terms of $\Lambda(t, c)$ there is no loss of generality assuming that $b \in \mathcal{D}_{d}$ is strictly decreasing on all of $\mathbb{R}_{+}$.

Proposition 4.4. Let $c=c(x)$ be constructed by $b \in \mathcal{D}_{d}$, cf. Definition 4.1. Then there exist functions $c_{1}$ and $c_{2}$, constructed by functions in $\mathcal{D}_{d}$ that are strictly decreasing on all of $\mathbb{R}_{+}$, such that $c_{1}(x) \leq c(x) \leq c_{2}(x), x \in \mathbb{R}^{d}$, and there exists $\rho \geq 0$ such that

$$
\begin{aligned}
& c_{1}(x)=c(x)=c_{2}(x), \quad|x| \geq \rho, \quad \text { if } c_{1}, c, c_{2} \text { are given as in }(\mathrm{C} 1), \\
& c_{1}(x)=c(x)=c_{2}(x), \quad \widehat{x} \geq \rho, \quad \text { if } c_{1}, c, c_{2} \text { are given as in }(\mathrm{C} 2) .
\end{aligned}
$$

As a result, there exists $\tau=\tau\left(c, c_{1}, c_{2}\right) \geq 0$ such that

$$
\Lambda(t, c)=\Lambda\left(t, c_{1}\right)=\Lambda\left(t, c_{2}\right), \quad t \geq \tau .
$$

Proof. Let $c$ be constructed by a $b \in \mathcal{D}_{d}$. By Definitions 2.6-2.7 there exists $\rho^{\prime}>0$ such that $b$ is decreasing on $\left(\rho^{\prime}, \infty\right)$ to 0 and, for some $D_{1}, D_{2}>0$, $D_{1} \leq b(s) \leq D_{2}$ for $s \in\left[0, \rho^{\prime}\right]$. Choose $\rho \geq \rho^{\prime}$ such that $b(\rho) \leq D_{1}$. Set $b_{1}(s)=$ $b_{2}(s)=b(s)$ for $s>\rho$ and define $b_{1}$ on $[0, \rho]$ as an arbitrary decreasing function with $b_{1}(0) \leq D_{1}$. Similarly, we define $b_{2}$ on $[0, \rho]$ as an arbitrary decreasing bounded function with $b_{2}(\rho) \geq D_{2}$. As a result, $b_{1}(s) \leq b(s) \leq b_{2}(s), s \in \mathbb{R}_{+}$. Let $c_{1}, c_{2}$ be constructed by $b_{1}, b_{2} \in \mathcal{D}_{d}$, which are strictly decreasing on $\mathbb{R}_{+}$ such that either $c_{1}, c_{2}, c$ are all given by $(\mathrm{C} 1)$ or $c_{1}, c_{2}, c$ are all given by $(\mathrm{C} 2)$. Then in both cases, evidently, $c_{1}(x) \leq c(x) \leq c_{2}(x), x \in \mathbb{R}^{d}$. The rest of the proof follows from Proposition 4.3.

Let $b, b^{\alpha} \in \mathcal{D}_{d}$ for some $\alpha \in(0,1)$. We denote by $c_{\alpha}$ the function constructed by $b^{\alpha}$, as in (3.7).

Remark 4.5. It is easy to see that, if for some $\alpha_{0} \in(0,1), b^{\alpha_{0}} \in \mathcal{D}_{d}$, then $b^{\alpha} \in \mathcal{D}_{d}$ for all $\alpha \in\left[\alpha_{0}, 1\right]$.

Proposition 4.6. For any $\alpha_{0} \in\left(\frac{3}{4}, 1\right)$ there exists $\varepsilon_{0}=\varepsilon_{0}\left(\alpha_{0}\right) \in(0,1)$ such that, for any $\varepsilon \in\left(0, \varepsilon_{0}\right)$, there exists $\alpha=\alpha(\varepsilon) \in\left(\alpha_{0}, 1\right)$ such that the following holds. For any $b \in \mathcal{D}_{d}$ strictly decreasing on $\mathbb{R}_{+}$such that $b^{\alpha_{0}} \in \mathcal{D}_{d}$, let $c$ and $c_{\alpha}$ be constructed by $b$ and $b^{\alpha}$, respectively. Then there exists $\tau=\tau(\varepsilon, b)>0$ such that, for any $t \geq \tau$,

$$
\begin{aligned}
& \Lambda\left(t-\varepsilon t, c_{\alpha}\right) \subset \Lambda\left(t-\frac{\varepsilon t}{2}, c\right), \\
& \Lambda\left(t+\frac{\varepsilon t}{2}, c_{\alpha}\right) \subset \Lambda(t+\varepsilon t, c) .
\end{aligned}
$$

Proof. Fix $\alpha_{0} \in\left(\frac{3}{4}, 1\right)$ and take an arbitrary $b \in \mathcal{D}_{d}$ such that $b^{\alpha_{0}} \in \mathcal{D}_{d}$; let $c$ be constructed by $b$. We will prove (4.3). The proof of (4.2) is fully analogous. Consider two cases (C1) and (C2) separately.

1) Let $c$ be given by $(\mathrm{C} 1)$. Since $\alpha_{0} \in\left(\frac{3}{4}, 1\right)$, one can define $\varepsilon_{0}:=\frac{1-\alpha_{0}}{\alpha_{0}-\frac{1}{2}} \in$ $(0,1)$. Take an arbitrary $\varepsilon \in\left(0, \varepsilon_{0}\right)$, then one easily has that

$$
\alpha:=\frac{1+\frac{\varepsilon}{2}}{1+\varepsilon} \in\left(\alpha_{0}, 1\right),
$$


and hence

$$
\exp \left(-\left(1+\frac{\varepsilon}{2}\right) \beta t\right)=(\exp (-(1+\varepsilon) \beta t))^{\alpha}
$$

Since $b$ is decreasing on $\mathbb{R}_{+}$, we get from (1.11) that the equality holds in (4.3) for all $t \geq 0$.

2) Let $c$ be given by (C2). Prove the following inequality, which is equivalent to $(4.3)$,

$$
\mathbb{R}^{d} \backslash \Lambda(t+\varepsilon t, c) \subset \mathbb{R}^{d} \backslash \Lambda\left(t+\frac{\varepsilon t}{2}, c_{\alpha}\right), \quad t \geq \tau .
$$

Recall that the inclusion $x \in \mathbb{R}^{d} \backslash \Lambda(t+\varepsilon t, c)$ is equivalent to

$$
c(x)=\int_{\Delta(x)} b(|y|) d y<e^{-\beta(1+\varepsilon) t} .
$$

We will use Hölder's inequality to estimate $c_{\alpha}(x)$. It is easy to see that the function

$$
f(\alpha):=\alpha-\sqrt{\alpha(1-\alpha)}:\left(\frac{1}{2}, 1\right) \rightarrow(0,1)
$$

is increasing. We set $p:=p(\alpha):=\frac{1}{f(\alpha)}>1$ and $q:=q(\alpha):=\frac{1}{1-f(\alpha)}>1$. Then $\frac{1}{p}+\frac{1}{q}=1$ and, by (4.6), we have

$$
\begin{aligned}
c_{\alpha}(x) & =\int_{\Delta(x)} b(|y|)^{f(\alpha)+(\alpha-f(\alpha))} d y \\
& \leq\left(\int_{\Delta(x)} b(|y|)^{f(\alpha) p} d y\right)^{\frac{1}{p}}\left(\int_{\Delta(x)} b(|y|)^{(\alpha-f(\alpha)) q} d y\right)^{\frac{1}{q}} \\
& <e^{-\beta(1+\varepsilon) f(\alpha) t}\left(\int_{\Delta(x)} b(|y|)^{(\alpha-f(\alpha)) q} d y\right)^{\frac{1}{q}}
\end{aligned}
$$

To get the finiteness of the latter integral in (4.7), it is enough to have there $\alpha$ such that $\alpha_{0}<g(\alpha)<1$, where

$$
g(\alpha):=(\alpha-f(\alpha)) q(\alpha)=\frac{\sqrt{\alpha}}{\sqrt{\alpha}+\sqrt{1-\alpha}}, \quad \alpha \in\left(\frac{1}{2}, 1\right) .
$$

It is easy to see that $g:\left(\frac{1}{2}, 1\right) \rightarrow\left(\frac{1}{2}, 1\right)$ is increasing and $g(\alpha)<\alpha, \alpha \in$ $\left(\frac{1}{2}, 1\right)$. Note also that $g\left(\frac{9}{10}\right)=\frac{3}{4}$. As a result, for the given $\alpha_{0} \in\left(\frac{3}{4}, 1\right)$, there exists a unique $\alpha_{1} \in\left(\frac{9}{10}, 1\right)$ such that $\alpha_{0}=g\left(\alpha_{1}\right)<\alpha_{1}$. Hence, for any $\alpha \in$ $\left(\alpha_{1}, 1\right) \subset\left(\alpha_{0}, 1\right)$, one gets $g(\alpha)>g\left(\alpha_{1}\right)=\alpha_{0}$, and then $\int_{\mathbb{R}^{d}} b(|y|)^{g(\alpha)} d y<\infty$; in particular, the latter integral in (4.7) is finite.

Next, the function $h(\varepsilon)=\frac{1+\frac{\varepsilon}{2}}{1+\varepsilon}:(0,1) \rightarrow\left(\frac{3}{4}, 1\right)$ is decreasing; cf. (4.4). Therefore, there exists a unique $\varepsilon_{0} \in(0,1)$ such that $h\left(\varepsilon_{0}\right)=\alpha_{1}$; then we have $h:\left(0, \varepsilon_{0}\right) \rightarrow\left(\alpha_{1}, 1\right)$. Take and fix now an arbitrary $\varepsilon \in\left(0, \varepsilon_{0}\right)$. Since,

$$
f:\left(\alpha_{1}, 1\right) \rightarrow\left(f\left(\alpha_{1}\right), 1\right) \subset\left(\alpha_{1}, 1\right)=\left(h\left(\varepsilon_{0}\right), 1\right)
$$

is increasing (we used here that $f(\alpha)<\alpha$ ), there exists a unique $\alpha=\alpha(\varepsilon) \in$ $\left(\alpha_{1}, 1\right)$ such that

$$
f(\alpha)=h(\varepsilon)=\frac{1+\frac{\varepsilon}{2}}{1+\varepsilon} .
$$


Therefore, after $\varepsilon_{0}, \varepsilon, \alpha$ are chosen, by the above, $\int_{\mathbb{R}^{d}} b(|y|)^{g(\alpha)} d y<\infty$; therefore, there exists $r>0$ such that, for all $x \in \mathbb{R}^{d}$ with $\widehat{x}>r$,

$$
\int_{\Delta(x)} b(|y|)^{g(\alpha)} d y \leq 1 .
$$

The latter inequality together with (4.8) and (4.7) implies that

$$
c_{\alpha}(x) \leq e^{-\beta\left(1+\frac{\varepsilon}{2}\right) t},
$$

provided that $x \in \mathbb{R}^{d} \backslash \Lambda(t+\varepsilon t, c)$ (i.e. (4.6) holds) and $\widehat{x}>r$. In (4.6), $\widehat{x} \rightarrow \infty$ if and only if $t \rightarrow \infty$; cf. Remark 4.2. Therefore, there exists $\tau=\tau(r)=\tau(\varepsilon, b)>0$ such that $t \geq \tau$ in (4.6) implies $\widehat{x} \geq r$. As a result, for any $t \geq \tau$ and any $x \in \mathbb{R}^{d} \backslash \Lambda(t+\varepsilon t, c)$, one gets (4.9), that means that $x \in \mathbb{R}^{d} \backslash \Lambda\left(t+\frac{\varepsilon t}{2}, c_{\alpha}\right)$; i.e. (4.5) holds.

Proposition 4.7. Let $b_{1}, b_{2} \in \mathcal{D}_{d}$ be log-equivalent functions such that, for some $\alpha_{0} \in\left(\frac{3}{4}, 1\right), b_{i}^{\alpha_{0}} \in \mathcal{D}_{d}, i=1,2$. Let $c^{(i)}$ be constructed by $b_{i}, i=1,2$ (both satisfy simultaneously either $(\mathrm{C} 1)$ or $(\mathrm{C} 2))$. Then there exists $\varepsilon_{0}=\varepsilon_{0}\left(\alpha_{0}\right) \in(0,1)$ such that, for any $\varepsilon \in\left(0, \varepsilon_{0}\right)$, there exists $\tau=\tau(\varepsilon)>0$ such that, for any $t \geq \tau$,

$$
\begin{aligned}
& \Lambda\left(t-\varepsilon t, c^{(1)}\right) \subset \Lambda\left(t-\frac{\varepsilon t}{2}, c^{(2)}\right), \\
& \Lambda\left(t+\frac{\varepsilon t}{2}, c^{(1)}\right) \subset \Lambda\left(t+\varepsilon t, c^{(2)}\right) .
\end{aligned}
$$

Proof. We assume first that both $b_{1}$ and $b_{2}$ are strictly decreasing on $\mathbb{R}_{+}$. Let $\varepsilon_{0}$ be given by Proposition 4.6. Take an arbitrary $\varepsilon \in\left(0, \varepsilon_{0}\right)$ and consider $\alpha=\alpha(\varepsilon) \in\left(\alpha_{0}, 1\right)$ also given by Proposition 4.6. Let $\rho_{0}>0$ be such that $b_{i}\left(\rho_{0}\right) \leq 1, i=1,2$. Set $\delta:=1-\alpha \in\left(0,1-\alpha_{0}\right)$. By (2.8), there exists $\rho_{\alpha} \geq \rho_{0}$ such that

in particular,

$$
1-\delta<\frac{-\log b_{1}(s)}{-\log b_{2}(s)}<1+\delta, \quad s>\rho_{\alpha},
$$

$$
b_{1}(s)<b_{2}(s)^{\alpha}, \quad s>\rho_{\alpha} .
$$

By Remark 4.5, $b_{2}^{\alpha} \in \mathcal{D}_{d}$, and hence, by (4.12) and Proposition 4.3, applying to $b_{1}$ and $b_{2}^{\alpha}$, one gets

$$
\Lambda\left(t+\frac{\varepsilon t}{2}, c^{(1)}\right) \subset \Lambda\left(t+\frac{\varepsilon t}{2}, c_{\alpha}^{(2)}\right) .
$$

The latter inequality together with (4.3) for $c=c^{(2)}$ imply (4.11).

Next, by $(4.12), b_{3}(s):=b_{1}(s)^{\frac{1}{\alpha}}<b_{2}(s)$, if only $s>\rho_{\alpha}$. From here we have that $b_{3} \in \mathcal{D}_{d}$ and, moreover, by Proposition 4.3, applying to $b_{3}$ and $b_{2}$,

$$
\Lambda\left(t-\frac{\varepsilon t}{2}, c^{(3)}\right) \subset \Lambda\left(t-\frac{\varepsilon t}{2}, c^{(2)}\right),
$$

where $c^{(3)}$ in constructed by $b_{3}$. The latter inequality together with (4.2) for $c=c^{(3)}$ imply (4.10).

Let now $b_{i} \in \mathcal{D}_{d}, i=1,2$ be arbitrary. Then, by the proof of Proposition 4.4, there exist $\tilde{c}^{(i)}$ constructed by $\tilde{b}_{i} \in \mathcal{D}_{d}$, strictly decreasing on $\mathbb{R}_{+}$such that $b_{i}(s)=\tilde{b}_{i}(s)$ for big enough $s$. Then $\tilde{b}_{1}$ and $\tilde{b}_{2}$ are log-equivalent. Applying the previous considerations to $\tilde{b}_{i}, i=1,2$, we get (4.10) and (4.11), with $c^{(i)}$ replaced by $\tilde{c}^{(i)}, i=1,2$, for big enough $t$. Then, by Proposition 4.4, one gets the statement. 


\subsection{Functions constructed by long-tailed functions}

Recall that long-tailed functions were defined in Definition 2.6.

Proposition 4.8 ([33, Lemma 4.1]). Let c given by (C1) be constructed by a long-tailed function $b \in \mathcal{D}_{d}$. Then, for any $r>0$,

$$
\lim _{|x| \rightarrow \infty} \sup _{|y| \leq r}\left|\frac{c(x+y)}{c(x)}-1\right|=0 .
$$

Proposition 4.9. Let c given by (C2) be constructed by a long-tailed function $b \in \mathcal{D}_{d}$. Then

$$
\lim _{\widehat{x} \rightarrow \infty} \frac{c(x+h)}{c(x)}=1, \quad h \in \mathbb{R}_{+}^{d} .
$$

Proof. Fix any $h \in \mathbb{R}_{+}^{d}$. Take an $\varepsilon \in(0,1)$. Since $b$ is long-tailed, we have that, for each $R \geq \widehat{h}$, there exists $s_{0}=s_{0}(\varepsilon, R)>0$, such that

$$
1-\varepsilon \leq \frac{b(\tau)}{b(s)} \leq 1+\varepsilon, \quad|\tau-s| \leq R, s \geq s_{0},
$$

because of e.g. [35, formula (2.18)]; cf. also [33, Remark 2.1].

Let $\widehat{x} \geq s_{0}(\varepsilon, R)$. We have that

$$
1-\frac{c(x+h)}{c(x)}=\frac{\int_{\Delta(x) \backslash \Delta(x+h)} b(|y|) d y}{\int_{\Delta(x)} b(|y|) d y} \geq 0 ;
$$

and

$$
\Delta(x) \backslash \Delta(x+h)=\left\{y \in \Delta(x) \mid \exists j: y_{j}<x_{j}+h_{j}\right\} \subset \bigcup_{j=1}^{d} \Delta_{j}(x),
$$

where

$$
\Delta_{j}(x):=\left\{y \in \Delta(x) \mid y_{j} \leq x_{j}+h_{j}\right\} .
$$

Set, for each $y=\left(y_{1}, \ldots, y_{d}\right) \in \Delta(x)$,

$$
y_{x_{j}}:=\left(y_{1}, \ldots, y_{j-1}, x_{j}, y_{j+1}, \ldots, y_{d}\right) .
$$

Note that, for each $y \in \Delta_{j}(x)$,

$$
|| y_{x_{j}}|-| y|| \leq\left|y_{x_{j}}-y\right|=\left|x_{j}-y_{j}\right| \leq \widehat{h} \leq R .
$$

Moreover, $\left|y_{x_{j}}\right| \geq \widehat{y_{x_{j}}} \geq \widehat{x} \geq s_{0}(\varepsilon, R)$. Then, by (4.15),

$$
\int_{\Delta_{j}(x)} b(|y|) d y=\int_{\Delta_{j}(x)} \frac{b(|y|)}{b\left(\left|y_{x_{j}}\right|\right)} b\left(\left|y_{x_{j}}\right|\right) d y \leq B_{x_{j}}(1+\varepsilon) \widehat{h},
$$

where

$$
\begin{aligned}
B_{x_{j}} & :=\int_{x_{1}}^{\infty} \ldots \int_{x_{j-1}}^{\infty} \int_{x_{j+1}}^{\infty} \ldots \int_{x_{d}}^{\infty} b\left(\left|y_{x_{j}}\right|\right) d y_{x_{j}} \in(0, \infty) \\
d y_{x_{j}} & :=d y_{1} \ldots d y_{j-1} d y_{j+1} \ldots d y_{d}
\end{aligned}
$$


On the other hand, for each $1 \leq j \leq d$, we get, by the same arguments

$$
\int_{\Delta(x)} b(|y|) d y \geq \int_{\left\{y \in \Delta(x) \mid y_{j} \leq x_{j}+R\right\}} \frac{b(|y|)}{b\left(\left|y_{x_{j}}\right|\right)} b\left(\left|y_{x_{j}}\right|\right) d y \geq B_{x_{j}}(1-\varepsilon) R .
$$

As a result,

$$
0 \leq 1-\frac{c(x+h)}{c(x)} \leq \sum_{j=1}^{d} \frac{B_{x_{j}}(1+\varepsilon) \widehat{h}}{B_{x_{j}}(1-\varepsilon) R}<\varepsilon,
$$

provided that $R=R(\widehat{h}, \varepsilon) \geq \widehat{h}$ is chosen big enough and $\widehat{x} \geq s_{0}(\varepsilon, R)$. The statement is proved.

Remark 4.10. Note that the previous result remains true if $c$ is defined by $(\mathrm{C} 2)$ with $\Delta(x)$ replaced by $\Delta\left(x+x_{0}\right)$ for a fixed $x_{0} \in \mathbb{R}^{d}$.

The following proposition gives a sufficient condition for (3.13); the result is a generalization of [35, Theorem 4.2].

Proposition 4.11. Let $f \in L^{1}\left(\mathbb{R}^{d} \rightarrow \mathbb{R}_{+}\right)$and $c: \mathbb{R}^{d} \rightarrow(0, \infty)$ be a bounded function such that (4.13) holds. Then

$$
\liminf _{|x| \rightarrow \infty} \frac{(c * f)(x)}{c(x)} \geq \int_{\mathbb{R}^{d}} f(y) d y .
$$

Moreover, there exists $D>0$ such that

$$
(c * f)(x) \geq D c(x), \quad x \in \mathbb{R}^{d} .
$$

Proof. For any $r>0$, we have

$$
\frac{(c * f)(x)}{c(x)} \geq\left(1-\sup _{|y| \leq r}\left|\frac{c(x-y)}{c(x)}-1\right|\right) \int_{|y| \leq r} f(y) d y .
$$

Take an arbitrary $\delta \in(0,1)$ and choose $r=r(\delta)>0$ such that $\int_{|y| \leq r} f(y) d y>$ $(1-\delta) \int_{\mathbb{R}^{d}} f(y) d y$. Next, by (4.13), there exists $\rho=\rho(r)=\rho(\delta) \geq r$ such that $\sup _{|y| \leq r}\left|\frac{c(x-y)}{c(x)}-1\right|<\delta$, for all $|x| \geq \rho$. As a result, for any $\delta \in(0,1)$, there exists $\rho=\rho(\delta)>0$ such that

$$
\frac{(c * f)(x)}{c(x)}>(1-\delta)^{2} \int_{\mathbb{R}^{d}} f(y) d y, \quad|x| \geq \rho,
$$

that yields (4.16). Finally, by e.g. [28, Lemma 2.1], $c * f$ is a continuous function on $B_{\rho}(0)$; then, it is easy to see that $c(x)>0, x \in \mathbb{R}^{d}$ implies that $(c * f)(x)>0$, $x \in \mathbb{R}^{d}$. Hence the boundedness of $c$ yields $\inf _{|x| \leq \rho} \frac{(c * f)(x)}{c(x)}>0$, that fulfilled the statement.

\section{Proofs}

In this Section, $\beta>0$ is given by (A1). 


\subsection{Proofs of upper estimates}

In this Subsection, we are going to prove Theorem 3.1.

\subsubsection{Preliminaries}

For a function $\widetilde{\omega}: \mathbb{R}^{d} \rightarrow(0,+\infty)$, we define, for any $f: \mathbb{R}^{d} \rightarrow \mathbb{R}$,

$$
\|f\|_{\widetilde{\omega}}:=\sup _{x \in \mathbb{R}^{d}} \frac{|f(x)|}{\widetilde{\omega}(x)} \in[0, \infty] .
$$

If $\widetilde{\omega}(x)=b(|x|), x \in \mathbb{R}^{d}$, for a function $b: \mathbb{R}_{+} \rightarrow(0, \infty)$, we will use the notation $\|f\|_{b}:=\|f\|_{\widetilde{\omega}}$.

Proposition 5.1 (cf. [30, Propostion 3.1]). Let a function $\widetilde{\omega}: \mathbb{R}^{d} \rightarrow(0,+\infty)$ be such that $a * \widetilde{\omega}$ is well-defined (for example, let $\widetilde{\omega}$ be bounded) and, for some $\gamma \in(0, \infty)$

$$
\frac{(a * \widetilde{\omega})(x)}{\widetilde{\omega}(x)} \leq \gamma, \quad x \in \mathbb{R}^{d}
$$

Let $0 \leq u_{0} \in L^{\infty}\left(\mathbb{R}^{d}\right)$ and $\left\|u_{0}\right\|_{\widetilde{\omega}}<\infty$; let $w=w(x, t)$ be the corresponding solution to (3.1). Then

$$
\|w(\cdot, t)\|_{\widetilde{\omega}} \leq\left\|u_{0}\right\|_{\widetilde{\omega}} e^{(\gamma-1+\beta) t}, \quad t \geq 0 .
$$

Proof. The solution to (3.1) is given by

$$
w(x, t)=e^{-m t} u_{0}(x)+e^{-m t} \sum_{n=1}^{\infty} \frac{t^{n}}{n !}\left(a^{* n} * u_{0}\right)(x),
$$

for $x \in \mathbb{R}^{d}, t \geq 0$, where $a^{* n}:=a * \ldots * a$ (the convolution is taken $n-1$ times). Since $\left\|u_{0}\right\|_{\widetilde{\omega}}<\infty$, we have $0 \leq u_{0}(x) \leq\left\|u_{0}\right\|_{\widetilde{\omega}} \widetilde{\omega}(x), x \in \mathbb{R}^{d}$. Next, (5.2) evidently implies

$$
\left(a^{* n} * \widetilde{\omega}\right)(x) \leq \gamma^{n} \widetilde{\omega}(x), \quad x \in \mathbb{R}^{d} .
$$

As a result, we get from (5.4) that

$$
\begin{aligned}
0 \leq w(x, t) & \leq e^{-m t}\left\|u_{0}\right\|_{\widetilde{\omega}} \widetilde{\boldsymbol{\omega}}(x)+e^{-m t}\left\|u_{0}\right\|_{\widetilde{\omega}} \sum_{n=1}^{\infty} \frac{t^{n}}{n !} \gamma^{n} \widetilde{\boldsymbol{\omega}}(x) \\
& =\left\|u_{0}\right\|_{\widetilde{\omega}} \widetilde{\boldsymbol{\omega}}(x)\left(e^{-m t}+e^{-m t}\left(e^{\gamma t}-1\right)\right)=\left\|u_{0}\right\|_{\widetilde{\omega}} \widetilde{\omega}(x) e^{(\gamma-m) t},
\end{aligned}
$$

that implies (5.3).

Remark 5.2. In [30, Propostion 3.1], we considered, for an arbitrary $\lambda>0$ and a unit vector, $\xi \in \mathbb{R}^{d}$, the function $\widetilde{\omega}(x)=e^{-\lambda x \cdot \xi}$ (recall that $x \cdot \xi$ stands for the scalar product in $\left.\mathbb{R}^{d}\right)$. Then, clearly, $\frac{\left(a^{+} * \widetilde{\boldsymbol{\omega}}\right)(x)}{\widetilde{\boldsymbol{\omega}}(x)} \equiv \int_{\mathbb{R}^{d}} a^{+}(y) e^{\lambda y \cdot \xi} d y=: \gamma$, provided that the latter integral is finite (that was the crucial assumption to get the constant speed of the front in [30]). Note that then [29, Proposition 2.4] and (5.3) implies that $w(x, t) \leq \alpha_{\xi} e^{\beta_{\xi} t-\lambda_{\xi} x \cdot \xi}, x \in \mathbb{R}^{d}, t \geq 0$ for some $\alpha_{\xi}, \lambda_{\xi}>0$, $\beta_{\xi} \in \mathbb{R}$. 
Proposition 5.3 ([33, Proposition 2.4]). Let a function $\omega: \mathbb{R}^{d} \rightarrow(0,+\infty)$ be such that, for any $\lambda>0$,

$$
\{\omega<\lambda\} \neq \varnothing,
$$

cf. (3.9). Suppose further that

$$
\eta:=\limsup _{\lambda \rightarrow 0+} \sup _{x \in\{\omega<\lambda\}} \frac{(a * \omega)(x)}{\omega(x)} \in(0, \infty) .
$$

Then, for any $\delta \in(0,1)$, there exists $\lambda=\lambda(\delta, \omega) \in(0,1)$ such that $(5.2)$ holds, with

$$
\widetilde{\omega}(x):=\omega_{\lambda}(x):=\min \{\lambda, \omega(x)\}, \quad x \in \mathbb{R}^{d},
$$

and $\gamma:=\max \{1,(1+\delta) \eta\}$.

Proposition 5.4. Let $\omega$ be constructed by $b \in \mathcal{D}_{d}$ (see Definition 4.1) and satisfy, cf. (5.6),

$$
\limsup _{\lambda \rightarrow 0+} \sup _{x \in\{\omega<\lambda\}} \frac{(a * \omega)(x)}{\omega(x)} \leq 1 .
$$

Let $0 \leq u_{0} \in L^{\infty}\left(\mathbb{R}^{d}\right)$ be such that $\left\|u_{0}\right\|_{\omega}<\infty$, cf. (5.1), and let $w=w(x, t)$ be the corresponding solution to (3.1). Then, for any $\varepsilon \in(0,1)$, there exist $A_{\varepsilon}>0$ and $t_{0}=t_{0}(\varepsilon)>0$ such that

$$
\operatorname{esssup}_{x \notin \Lambda(t+\varepsilon t, \omega)} w(x, t) \leq\left(A_{\varepsilon}+\left\|u_{0}\right\|_{\omega}\right) e^{-\frac{\varepsilon \beta}{2} t}, \quad t \geq t_{0} .
$$

Proof. Take an arbitrary $\varepsilon \in(0,1)$ and let $\delta=\delta(\varepsilon) \in(0,1)$ be chosen later. By Proposition 3.2, condition (5.6) holds. Then, by Proposition 5.3, there exists $\lambda=\lambda(\delta, \omega)=\lambda(\varepsilon, \omega) \in(0,1)$ such that $(5.2)$ holds, with $\widetilde{\omega}$ given by $(5.7)$ and $\gamma=1+\delta$. Set $\left\|u_{0}\right\|_{\infty}:=\left\|u_{0}\right\|_{L^{\infty}\left(\mathbb{R}^{d}\right)}$. Note that

$$
\frac{u_{0}(x)}{\omega_{\lambda}(x)} \leq \frac{1}{\lambda} \mathbb{1}_{\mathbb{R}^{d} \backslash\{\omega<\lambda\}}(x)+\frac{u_{0}(x)}{\omega(x)} \mathbb{1}_{\{\omega<\lambda\}}(x) \leq \frac{\left\|u_{0}\right\|_{\infty}}{\lambda}+\left\|u_{0}\right\|_{\omega}<\infty,
$$

and one can apply Proposition 5.1. Namely, setting $A_{\varepsilon}:=\frac{\left\|u_{0}\right\|_{\infty}}{\lambda}>0$, one gets from (5.10), (5.3) that, for a.a. $x \in\{\omega<\lambda\}$ and for all $t \geq 0$,

$$
w(x, t) \leq\left\|u_{0}\right\|_{\omega_{\lambda}} e^{(\delta+\beta) t} \omega_{\lambda}(x) \leq\left(A_{\varepsilon}+\left\|u_{0}\right\|_{\omega}\right) e^{(\delta+\beta) t} \omega(x) .
$$

By (5.5),

$$
\mathbb{R}^{d} \backslash \Lambda(t+\varepsilon t, \omega)=\left\{\omega<e^{-\beta(t+\varepsilon t)}\right\}, \quad t>0 .
$$

Set $t_{0}=t_{0}(\varepsilon):=-\frac{1}{(1+\varepsilon) \beta} \log \lambda>0$. One gets from (5.12) that, for any $t \geq t_{0}$,

$$
\mathbb{R}^{d} \backslash \Lambda(t+\varepsilon t, \omega) \subset \mathbb{R}^{d} \backslash \Lambda\left(t_{0}+\varepsilon t_{0}, \omega\right)=\{\omega<\lambda\} .
$$

Hence, by (5.11), (5.12), for a.a. $x \in \mathbb{R}^{d} \backslash \Lambda(t+\varepsilon t, \omega)$, one gets

$$
w(x, t) \leq\left(A_{\varepsilon}+\left\|u_{0}\right\|_{\omega}\right) e^{(\delta+\beta) t} \boldsymbol{\omega}(x) \leq\left(A_{\varepsilon}+\left\|u_{0}\right\|_{\omega}\right) e^{(\delta+\beta) t} e^{-\beta(t+\varepsilon t)},
$$

and

$$
\delta+\beta-\beta(1+\varepsilon)=\delta-\beta \varepsilon=-\frac{\varepsilon \beta}{2},
$$

if only we set from the very beginning $\delta:=\frac{\varepsilon \beta}{2}$. The statement is proved. 
Remark 5.5. It is easy to see from the proof above, that the denominator 2 in the right-hand side of (5.9) can be changed on $1+\nu$, for an arbitrary $\nu \in(0,1)$; then $t_{0}=t_{0}(\varepsilon, \nu)$.

\subsubsection{Proof of Proposition 3.2}

We are going to show now that for the functions $\omega=c_{\alpha}$ from (3.7) the inequality (5.8) holds.

Case 1. $c_{\alpha}(x)=b(|x|)^{\alpha}$.

Proposition 5.6. Let $b \in \mathcal{E}_{d}$. Then there exists $\alpha_{1} \in(0,1)$ such that, for all $\alpha \in\left[\alpha_{1}, 1\right], b^{\alpha} \in \mathcal{E}_{d}$.

Proof. Let $d>1$ and $b \in \mathcal{E}_{d}$. If $b$ is given by (2.6), then, for any $\alpha^{\prime} \in\left(\frac{d}{d+\mu}, 1\right)$,

$$
\int_{0}^{\infty} b(s)^{\alpha^{\prime}} s^{d-1} d s<\infty .
$$

If $b$ is such that, for all $\nu \geq 1,(2.7)$ holds, then, evidently, (5.13) holds for all $\alpha^{\prime} \in(0,1)$. For $d=1$ and $b \in \mathcal{E}_{1},(5.13)$ holds if only $\alpha^{\prime} \in\left(\frac{1}{1+\delta}, 1\right)$, where $\delta$ is sufficiently small. Then, by [33, Theorem 3.1], there exists $\alpha_{1} \in\left(\alpha^{\prime}, 1\right)$ such that, for all $\alpha \in\left[\alpha_{0}, 1\right], b^{\alpha} \in \mathcal{E}_{d}$. The proof is fulfilled.

The following proposition ensures that (5.8) holds for $\omega=c_{\alpha}$ given as in (C1).

Proposition 5.7 ([33, Propositions 4.2, 4.3]). Let (3.3) hold with $b_{1} \in \mathcal{D}_{d}$ which is strictly decreasing on $\mathbb{R}_{+}$and log-equivalent to a function $b \in \mathcal{E}_{d}$. Then there exists $\alpha_{1} \in(0,1)$ such that, for all $\alpha \in\left(\alpha_{1}, 1\right)$, the function $\omega(x)=b(|x|)^{\alpha}$, $x \in \mathbb{R}^{d}$, satisfies (5.8).

Case 2. $c_{\alpha}(x)=\int_{\Delta(x)} b(|y|)^{\alpha} d y$.

Now we will show that (5.8) holds for $\omega=c_{\alpha}$ given as in (C2). Firstly, we prove that (5.8) holds with $\{\omega<\lambda\}$ replaced by the set $\Theta_{\lambda}(p)$ defined below and then we prove that the former set is a subset of the latter one for small enough $\lambda$. We start hence with the following definition.

Definition 5.8. Let $p(x)=b(|x|)$, for $b \in \mathcal{D}_{d}$. For any $\lambda \in(0, b(0))$, we set

$$
\Theta_{\lambda}(p):=\left\{x \in \mathbb{R}^{d}: \Delta(x) \subset\{p<\lambda\}\right\}
$$

where $\Delta(x)$ is given by (1.15) and $\{p<\lambda\}$ is defined as in (3.9).

Proposition 5.9. Let $p(x)=b(|x|)$, for $b \in \mathcal{D}_{d}$. Suppose that (5.8) holds with $\omega=p$. Let $c$ be given by (C2). Then the following analogue to (5.8) holds:

$$
\limsup _{\lambda \rightarrow 0+} \sup _{x \in \Theta_{\lambda}(p)} \frac{(a * c)(x)}{c(x)} \leq 1 .
$$


Proof. By Proposition 4.4, there is no loss of generality in assuming that $b$ is strictly decreasing on $\mathbb{R}_{+}$. Take an arbitrary $\delta \in(0,1)$. By $(5.8)$ with $\omega=p$, there exists $\lambda_{0}=\lambda_{0}(\delta)$ such that, for all $\lambda \in\left(0, \lambda_{0}\right)$, we have

$$
\frac{(a * p)(x)}{p(x)} \leq 1+\delta, \quad x \in\{p<\lambda\} .
$$

Next, for any $x \in \mathbb{R}^{d}$, one gets, cf. (3.16),

$$
\begin{aligned}
(a * c)(x) & =\int_{\mathbb{R}^{d}} a(x-y) \int_{\Delta(y)} p(z) d z d y \\
& =\int_{\mathbb{R}^{d}} a(x-y) \int_{\Delta(x)} p(z-(x-y)) d z d y=\int_{\Delta(x)}(a * p)(z) d z
\end{aligned}
$$

As a result, by (5.16) and (5.14), we have that, for any $x \in \Theta_{\lambda}(p)$,

$$
\frac{(a * c)(x)}{c(x)}=\frac{1}{c(x)} \int_{\Delta(x)} \frac{(a * p)(z)}{p(z)} p(z) d z \leq 1+\delta .
$$

Since the latter holds for any $\lambda \in\left(0, \lambda_{0}\right)$, one gets the statement.

To get from (5.15) the inequality (5.8) with $\omega=c$, we consider the following statement.

Proposition 5.10. Let $p(x)=b(|x|), x \in \mathbb{R}^{d}$, for a long-tailed function $b \in \mathcal{D}_{d}$. Let $c$ be given by (C2). Then there exists $\lambda_{1}>0$ such that, for all $\lambda \in\left(0, \lambda_{1}\right)$,

$$
\{c<\lambda\} \subset \Theta_{\lambda}(p) .
$$

Proof. By Proposition 4.4, there is no loss of generality in assuming that $b$ is strictly decreasing on $\mathbb{R}_{+}$. By Proposition 4.8 , we have that (4.13) holds with $c$ replaced by $p$. As a result, for any $\varepsilon>0$ and $r>0$, there exists $R=R(\varepsilon, r)>0$ such that

$$
p(x+y) \geq(1-\varepsilon) p(x), \quad|y| \leq r,|x| \geq R .
$$

Therefore, $x \in\{c<\lambda\}$ with $|x| \geq R$ implies that

$$
\begin{aligned}
\lambda & \geq \int_{x_{1}}^{x_{1}+\frac{r}{\sqrt{d}}} \ldots \int_{x_{d}}^{x_{d}+\frac{r}{\sqrt{d}}} b\left(\sqrt{y_{1}^{2}+\ldots+y_{d}^{2}}\right) d y_{1} \ldots d y_{d} \\
& \geq \frac{r^{d}}{d^{\frac{d}{2}}} p\left(x+\left(\frac{r}{\sqrt{d}}, \ldots, \frac{r}{\sqrt{d}}\right)\right) \geq \frac{r^{d}}{d^{\frac{d}{2}}}(1-\varepsilon) p(x) .
\end{aligned}
$$

Choose now any $\varepsilon \in\left(0, \frac{1}{2}\right)$ and $r=2^{\frac{1}{d}} \sqrt{d}>0$, and consider the corresponding $R$. Since $\lambda \downarrow 0$ if and only if $\widehat{x} \rightarrow \infty$, there exists $\lambda_{1}>0$ such that, for all $\lambda \in\left(0, \lambda_{1}\right)$, the inclusion $x \in\{c<\lambda\}$ implies $\widehat{x}>R$ and hence $|x|>R$. Moreover, for any $y \in \Delta(x)$, we have that $y \in\{c<\lambda\}$, by the monotonicity of $c$ in each of variables; and also we have that $\widehat{x}>R$ implies $|y|>R$. As a result, for any $y \in \Delta(x)$ (including $y=x$ ), we have that $p(y)<\lambda$, i.e $\Delta(x) \subset\{p<\lambda\}$. Then, by (5.14), $x \in \Theta_{\lambda}(p)$, that proves the statement.

Combination of Propositions 5.6, 5.7, 5.9, 5.10 evidently implies Proposition 3.2 . 


\subsubsection{Proof of Theorem 3.1}

Proof of Theorem 3.1. Let $b_{1}, b_{2} \in \mathcal{D}_{d}$ and $b \in \mathcal{E}_{d}$ satisfy the conditions of Theorem 3.1, and let $c$ and $c_{2}$ be constructed by $b$ and $b_{2}$, respectively (both are defined simultaneously by either $(\mathrm{C} 1)$ or $(\mathrm{C} 2)$ ). By Proposition 4.4, there is no loss of generality in assuming that all functions $b_{1}, b_{2}, b$ are strictly decreasing on $\mathbb{R}_{+}$.

By Propositions 5.6-5.7, there exists $\alpha_{1} \in(0,1)$ such that, for all $\alpha \in\left[\alpha_{1}, 1\right]$, $b^{\alpha} \in \mathcal{E}_{d}$, and for all $\alpha \in\left(\alpha_{1}, 1\right)$, the function $\omega(x)=b(|x|)^{\alpha}, x \in \mathbb{R}^{d}$, satisfies (5.8). Choose any $\alpha_{0} \in\left(\max \left\{\alpha_{1}, \frac{3}{4}\right\}, 1\right)$. Let $\varepsilon_{0}=\varepsilon_{0}\left(\alpha_{0}\right)$ be given by Proposition 4.6. Take an arbitrary $\varepsilon \in\left(0, \varepsilon_{0}\right)$ and consider $\alpha=\alpha(\varepsilon) \in\left(\alpha_{0}, 1\right)$ also given by Proposition 4.6. Since $\log b(s) \sim \log b_{2}(s), s \rightarrow \infty$, there exists $\rho=\rho(\alpha)=\rho(\varepsilon)>0$ such that

$$
-\log b_{2}(s) \geq-\alpha \log b(s)>0, \quad s>\rho .
$$

Therefore, $b_{2}(s) \leq b(s)^{\alpha}$ for $s>\rho$, and since both functions $b_{2}$ and $b$ are decreasing and separated from 0 on $[0, \rho]$, there exists $B>0$ such that $b_{2}(s) \leq$ $B b(s)^{\alpha}, s \in \mathbb{R}_{+}$. Let $c_{\alpha}$ be given by (3.7). Then, clearly, $c_{2}(x) \leq B c_{\alpha}(x), x \in \mathbb{R}^{d}$. As a result,

$$
\left\|u_{0}\right\|_{c_{\alpha}} \leq \frac{1}{B}\left\|u_{0}\right\|_{c_{2}}<\infty
$$

If $c$ is given by (C1), then by the assumed, (5.8) holds for $\omega=c_{\alpha}$ (see (3.7)).

Let now $c$ be given by (C2). Since $b$ is long-tailed, the function $b^{\alpha}$ is longtailed as well. Then, one can use Proposition 5.10 with $p$ replaced by $b^{\alpha}$; one gets then, for some $\lambda_{1}>0$,

$$
\left\{c_{\alpha}<\lambda\right\} \subset \Theta_{\lambda}\left(p^{\alpha}\right), \quad \lambda \in\left(0, \lambda_{1}\right) .
$$

Therefore, Proposition 5.9 implies that (5.8) holds for $\omega=c_{\alpha}$.

As a result, one can use now Proposition 5.4 with $\omega=c_{\alpha}$ and $\varepsilon$ replaced by $\frac{\varepsilon}{2}$. Namely, there exist $A_{\varepsilon}>0$ and $t_{0}=t_{0}(\varepsilon)>0$ such that

$$
\operatorname{ess~sup}_{x \notin \Lambda\left(t+\frac{\varepsilon t}{2}, c_{\alpha}\right)} w(x, t) \leq\left(A_{\varepsilon}+B^{-1}\left\|u_{0}\right\|_{c_{2}}\right) e^{-\frac{\varepsilon \beta}{4} t}, \quad t \geq t_{0} .
$$

On the other hand, by Proposition 4.6, there exists $\tau=\tau(\varepsilon)>t_{0}$ such that (4.3) holds, i.e.

$$
\mathbb{R}^{d} \backslash \Lambda(t+\varepsilon t, c) \subset \mathbb{R}^{d} \backslash \Lambda\left(t+\frac{\varepsilon t}{2}, c_{\alpha}\right), \quad t \geq \tau .
$$

Combining (5.17) and (5.18), one gets (3.6).

\subsection{Proofs of lower estimates}

In this Subsection, we are going to prove Theorem 3.3.

Let $c$ given by $b \in \mathcal{D}_{d}$ be fixed (see Definition 4.1). For any $\lambda>0$, we define the following function, for $x \in \mathbb{R}^{d}, t \geq 0$,

$$
\begin{aligned}
g(x, t) & =g_{c, \varepsilon, \lambda}(x, t)=\lambda \min \left\{1, c(x) e^{\beta(t-\varepsilon t)}\right\} \\
& =\lambda \mathbb{1}_{\Lambda(t-\varepsilon t, c)}(x)+\lambda c(x) e^{\beta(t-\varepsilon t)} \mathbb{1}_{\mathbb{R}^{d} \backslash \Lambda(t-\varepsilon t, c)}(x) .
\end{aligned}
$$


Define, for any $\lambda>0, \varepsilon \in(0,1)$ and for $\eta(t)=b^{-1}\left(e^{-\beta t}\right)$,

$$
f(s, t):=\lambda \mathbb{1}_{s \leq \eta(t-\varepsilon t)}+\lambda e^{\beta(t-\varepsilon t)} b(s) \mathbb{1}_{s>\eta(t-\varepsilon t)} \in[0, \lambda],
$$

By Definition 2.7, for any $\varepsilon \in(0,1)$ there exists $\tilde{\tau}=\tilde{\tau}(\varepsilon)>0$ such that

$$
g(x, t)=f(|x|, t), \quad x \in \mathbb{R}^{d}, t \geq \tilde{\tau} .
$$

Proposition 5.11. Let $c=c(x)$ be given by (C1) with a long-tailed, tail-logconvex function $b \in \mathcal{D}_{d}$. Then, for any $\tau>0$, the function $f$ defined by (5.21) satisfies the limit

$$
\lim _{t \rightarrow \infty} \sup _{s \in \mathbb{R}_{+}}\left|\frac{f(s+\tau, t)}{f(s, t)}-1\right|=0 .
$$

Proof. Take an arbitrary $\varepsilon \in(0,1)$. For an arbitrary fixed $\tau \in \mathbb{R}_{+}$, redefine $\tilde{\tau}$ such that $\eta(\tilde{\tau}-\varepsilon \tilde{\tau}) \geq \tau$. Then, for any $t \geq \tilde{\tau}$, the function $F_{\tau, t}(s):=\frac{f(s+\tau, t)}{f(s, t)}$ takes the following values. For $0 \leq s \leq \eta(t-\varepsilon t)-\tau$, one has $F_{\tau, t}(s)=1$. For $\eta(t-\varepsilon t)-\tau<s \leq \eta(t-\varepsilon t)$, we have $F_{\tau, t}(s)=e^{\beta(t-\varepsilon t)} b(s+\tau)$ and, since $b$ is decreasing on $[\eta(t-\varepsilon t), \infty)$, one gets

$$
\begin{aligned}
\frac{b(\eta(t-\varepsilon t)+\tau)}{b(\eta(t-\varepsilon t))} & =e^{\beta(t-\varepsilon t)} b(\eta(t-\varepsilon t)+\tau) \\
& \leq e^{\beta(t-\varepsilon t)} b(s+\tau) \leq e^{\beta(t-\varepsilon t)} b(\eta(t-\varepsilon t))=1 .
\end{aligned}
$$

Finally, for $s>\eta(t-\varepsilon t)$, we have, $F_{\tau, t}(s)=\frac{b(s+\tau)}{b(s)} \leq 1$ (since $b$ is decreasing),

$$
\frac{b(s+\tau)}{b(s)} \geq \frac{b(\eta(t-\varepsilon t)+\tau)}{b(\eta(t-\varepsilon t))} .
$$

As a result, for all $s \in \mathbb{R}_{+}$,

$$
0 \leq 1-F_{\tau, t}(s) \leq \mathbb{1}_{\{s>\eta(t-\varepsilon t)-\tau\}}(s)\left(1-\frac{b(\eta(t-\varepsilon t)+\tau)}{b(\eta(t-\varepsilon t))}\right),
$$

that implies the statement because of (2.5).

Proposition 5.12. Let $c=c(x)$ be given by $(\mathrm{C} 2)$ with a long-tailed, tail-logconvex function $b \in \mathcal{D}_{d}$. Let $g$ be given by (5.20). Then, for any $h \in \mathbb{R}_{+}^{d}$,

$$
\lim _{t \rightarrow \infty} \sup _{x \in \mathbb{R}^{d}}\left|\frac{g(x+h, t)}{g(x, t)}-1\right|=0 .
$$

Proof. Take an arbitrary $x \in \mathbb{R}^{d}, h \in \mathbb{R}_{+}^{d}$ and $t \geq \tilde{\tau}$. It is easy to see that $x \in \mathbb{R}^{d} \backslash \Lambda(t-\varepsilon t, c)$ implies $x+h \in \mathbb{R}^{d} \backslash \Lambda(t-\varepsilon t, c)$, and hence by monotonicity,

$$
\frac{g(x+h, t)}{g(x, t)}=\frac{c(x+h)}{c(x)} \leq 1 .
$$

Let $x \in \Lambda(t-\varepsilon t, c)$. If $x+h \in \Lambda(t-\varepsilon t, c)$, then $\frac{g(x+h, t)}{g(x, t)}=1$. Let now $h$ be such that $x+h \in \mathbb{R}^{d} \backslash \Lambda(t-\varepsilon t, c)$. Then

$$
\frac{g(x+h, t)}{g(x, t)}=e^{\beta(t-\varepsilon t)} c(x+h) \leq 1 .
$$


Moreover, since $x \in \Lambda(t-\varepsilon t, c)$ implies $c(x) e^{\beta(t-\varepsilon t)} \geq 1$, one has for such $x, h$ the following estimate

$$
0 \leq 1-\frac{g(x+h, t)}{g(x, t)} \leq 1-\frac{c(x+h)}{c(x)}
$$

As a result,

$$
\left|\frac{g(x+h, t)}{g(x, t)}-1\right|=1-\frac{g(x+h, t)}{g(x, t)} \leq \sup _{y: c(y+h)<e^{-\beta(t-\varepsilon t)}}\left(1-\frac{c(y+h)}{c(y)}\right) .
$$

Because of (4.14), for the chosen $h \in \mathbb{R}_{+}^{d}$ and for an arbitrary $\delta>0$, there exists $\rho=\rho(\delta, h)>0$ such that $\sup _{1 \leq j \leq d} y_{j}>\rho$ implies

$$
0 \leq 1-\frac{c(y+h)}{c(y)} \leq \delta .
$$

Choose now $t_{0}=t_{0}(\rho, \varepsilon, h)=t_{0}(\delta, \varepsilon, h) \geq \tilde{\tau}$ such that $c((\rho, \ldots, \rho)+h)>$ $e^{-\beta\left(t_{0}-\varepsilon t_{0}\right)}$. Prove that then, for any $t \geq t_{0}$, the inequality $c(y+h) \leq e^{-\beta(t-\varepsilon t)}$ implies $\sup _{1 \leq j \leq d} y_{j}>\rho$. Indeed, on the contrary, suppose that, for some $t \geq t_{0}$, the inequality $c(y+h) \leq e^{-\beta(t-\varepsilon t)}$ holds, however, $\sup _{1 \leq j \leq d} y_{j} \leq \rho$. The latter yields

$$
e^{-\beta(t-\varepsilon t)} \geq c(y+h) \geq c((\rho, \ldots, \rho)+h)>e^{-\beta\left(t_{0}-\varepsilon t_{0}\right)},
$$

that contradicts to that $t \geq t_{0}$. As a result, for all $x \in \mathbb{R}^{d}$ and $t>t_{0}$,

$$
\left|\frac{g(x+h, t)}{g(x, t)}-1\right| \leq \sup _{y: \sup _{1 \leq j \leq d} y_{j}>\rho}\left(1-\frac{c(y+h)}{c(y)}\right)<\delta,
$$

that implies the statement.

Definition 5.13. Let (A1) hold. A function

$$
v \in C\left(\mathbb{R}^{d} \times[\tau, \infty) \rightarrow \mathbb{R}_{+}\right) \cap C^{1}\left(\mathbb{R}^{d} \times(\tau, \infty) \rightarrow \mathbb{R}_{+}\right)
$$

is said to be a sub-solution to $(3.1)$ on $[\tau, \infty)$ for some $\tau \geq 0$, if

$$
\left(\mathcal{F}_{m} v\right)(x, t):=\frac{\partial}{\partial t} v(x, t)-(a * v)(x, t)+m v(x, t) \leq 0
$$

for a.a. $x \in \mathbb{R}^{d}$ and for all $t \in[\tau, \infty)$.

Proposition 5.14. Let (A1) hold and $c=c(x)$ be constructed by a long-tailed, tail-log-convex $b \in \mathcal{D}_{d}$. Let, for $\varepsilon \in(0,1)$ and $\lambda>0$, the function $g=g(x, t)$ be given by (5.20). For a fixed $\sigma>0$, we define

$$
v(x, t)=v_{c, \varepsilon, \lambda}(x, t):=\frac{1}{\sigma} \int_{t}^{t+\sigma} g(x, s) d s .
$$

Then there exists $\tau_{0}=\tau_{0}(\varepsilon)>0$ such that $v$ is a sub-solution to $(3.1)$ on $\left[\tau_{0}, \infty\right)$. 
Proof. Firstly, note that

$$
\begin{aligned}
\frac{\partial}{\partial t} v(x, t) & =\frac{1}{\sigma}(g(x, t+\sigma)-g(x, t))=\frac{1}{\sigma} \int_{t}^{t+\sigma} \frac{\partial}{\partial s} g(x, s) d s, \\
(a * v)(x, t) & =\frac{1}{\sigma} \int_{t}^{t+\sigma}(a * g)(x, s) d s
\end{aligned}
$$

and hence

$$
\left(\mathcal{F}_{m} v\right)(x, t)=\frac{1}{\sigma} \int_{t}^{t+\sigma}\left(\mathcal{F}_{m} g\right)(x, s) d s .
$$

Therefore, since the mapping $t \mapsto v(\cdot, t) \in E$ is continuously differentiable for $t>\tilde{\tau}$, to prove that $v$ is a sub-solution to (3.1) it is enough to show that $\left(\mathcal{F}_{m} g\right)(x, s) \leq 0$ for a.a. $x \in \mathbb{R}^{d}$ and a.a. $t>\tilde{\tau}$.

We have

$$
\begin{aligned}
\frac{\partial}{\partial t} g(x, t) & =\lambda(\beta-\varepsilon \beta) e^{\beta(t-\varepsilon t)} b(|x|) \mathbb{1}_{|x|>\eta(t-\varepsilon t)} \\
& =(\beta-\varepsilon \beta) g(x, t) \mathbb{1}_{|x|>(t-\varepsilon t)} \leq(\beta-\varepsilon \beta) g(x, t) .
\end{aligned}
$$

Therefore, by (5.25), (5.27),

$$
-\left(\mathcal{F}_{m} g\right) \geq a * g-m g-\beta(1-\varepsilon) g=a * g-g+\beta \varepsilon g .
$$

To find now an appropriate bound from below for $L g=a * g-g$, cf. (1.2), consider two cases separately.

1. Let $c$ by given by (C1). Since $f$ given by (5.21) is decreasing in its first coordinate, we have

$$
\begin{aligned}
(a * g)(x, t) & =\int_{\mathbb{R}^{d}} a(-y) g(x+y, t) d y=\int_{\mathbb{R}^{d}} a(-y) f(|x+y|, t) d y \\
& \geq \int_{\mathbb{R}^{d}} a(-y) f(|x|+|y|, t) d y=\int_{\mathbb{R}^{d}} a(y) f(|x|+|y|, t) d y \\
& =g(x, t) \int_{\mathbb{R}^{d}} a(y) \frac{f(|x|+|y|, t)}{f(|x|, t)} d y
\end{aligned}
$$

for a.a. $x \in \mathbb{R}^{d}$. Note that, by $(5.23)$,

$$
0<\frac{f(|x|+|y|, t)}{f(|x|, t)} \leq 1, \quad x, y \in \mathbb{R}^{d}, t \geq \tilde{\tau} .
$$

By (5.29), (5.30), and $\int_{\mathbb{R}^{d}} a(x) d x=1$, we have, cf. (5.28),

$$
(a * g)(x, t)-g(x, t) \geq-g(x, t) \int_{\mathbb{R}^{d}} a(y)\left|\frac{f(|x|+|y|, t)}{f(|x|, t)}-1\right| d y .
$$

Next, by (5.22), (5.30), and the dominated convergence theorem, one gets

$$
\lim _{t \rightarrow \infty} \int_{\mathbb{R}^{d}} a(y) \sup _{x \in \mathbb{R}^{d}}\left|\frac{f(|x|+|y|, t)}{f(|x|, t)}-1\right| d y=0 .
$$

Therefore, for any $\delta \in(0,1)$ (small enough later), there exists a $\tau_{0} \geq \tilde{\tau}$ such that, for all $t \geq \tau_{0}$ and for a.a. $x \in \mathbb{R}^{d}$,

$$
(a * g)(x, t)-g(x, t) \geq-\delta g(x, t) .
$$


As a result, by (5.28),

$$
-\mathcal{F}_{m} g \geq-\delta g+\beta \varepsilon g \geq 0,
$$

if only $\delta<\beta \varepsilon$. The proof, for $c$ given by (C1), is fulfilled.

2. Let $c$ be given by (C2). Denote, for any $y \in \mathbb{R}^{d}$,

$$
y^{+}:=\left(\left|y_{1}\right|, \ldots,\left|y_{d}\right|\right) \in \mathbb{R}_{+}^{d} .
$$

Since the function $c$ is decreasing along all basis directions, we easily get that the function $g$ given by (5.20) has the same property (in $x$ ). Therefore, since $y_{j} \leq y_{j}^{+}, j=1, \ldots, d$, one gets

$$
g(x+y, t) \geq g\left(x+y^{+}, t\right) .
$$

Therefore, we will have, instead of (5.29),

$$
\begin{aligned}
(a * g)(x, t) & =\int_{\mathbb{R}^{d}} a(-y) g(x+y, t) d y \geq \int_{\mathbb{R}^{d}} a(-y) g\left(x+y^{+}, t\right) d y \\
& =g(x, t) \int_{\mathbb{R}^{d}} a(y)\left(\frac{g\left(x+y^{+}, t\right)}{g(x, t)}-1\right) d y+g(x, t) \int_{\mathbb{R}^{d}} a(y) d y
\end{aligned}
$$

Taking into account (5.24) for $h=y^{+}$, the rest of the proof is fully analogous to the first part.

Definition 5.15. A function $w: \mathbb{R}^{d} \times \mathbb{R}_{+} \rightarrow \mathbb{R}_{+}$is said to be a sub-solution to $(2.1)$ on $[\tau, \infty)$ for some $\tau \geq 0$, if $(\mathcal{F} w)(x, t) \leq 0$ for a.a. $x \in \mathbb{R}^{d}$ and for all $t \in[\tau, \infty)$, where $\mathcal{F}$ is given by, cf. (5.25),

$$
(\mathcal{F} u)(x, t):=\frac{\partial}{\partial t} u(x, t)-(a * u)(x, t)+m u(x, t)+u(x, t)(G u)(x, t) .
$$

The proof of the following statement follows directly from Theorem 2.2.

Proposition 5.16. Let (A1)-(A4) hold. Let $0 \leq u \leq 1$ be a solution to (2.1), and $v: \mathbb{R}^{d} \times \mathbb{R}_{+} \rightarrow \mathbb{R}_{+}$be a sub-solution to (2.1) on $[\tau, \infty)$ for some $\tau \geq 0$. Suppose that, for some $t_{0}, t_{1} \geq \tau$, we have $u\left(x, t_{0}\right) \geq v\left(x, t_{1}\right)$ for a.a. $x \in \mathbb{R}^{d}$. Then

$$
u\left(x, t+t_{0}\right) \geq v\left(x, t+t_{1}\right), \quad x \in \mathbb{R}^{d}, t \geq 0 .
$$

We are going to find now, using the continuity of $G$ at 0 (on $E^{+}$, cf. (A3)) and Proposition 5.14, sufficient conditions to have (5.20) as a sub-solution to (2.1) as well.

Proposition 5.17. Let (A1)-(A4) hold and $c=c(x)$ be constructed by a longtailed, tail-log-convex function $b \in \mathcal{D}_{d}$. Then, for any $\varepsilon \in(0,1)$, there exist $\lambda_{0}=\lambda_{0}(\varepsilon)>0$ and $\tau_{0}=\tau_{0}(\varepsilon)>0$, such that, for any $\lambda \in\left[0, \lambda_{0}\right]$, the function $v=v(x, t)$, given by (5.26) and (5.20), is a sub-solution to $(2.1)$ on $\left[\tau_{0}, \infty\right)$.

Proof. Take an arbitrary $\varepsilon \in(0,1)$. For any $\delta \in(0, \varepsilon \beta)$, one has that $m+\delta<$ $m+\beta=1$; hence one can apply Proposition 5.14 to the equation (3.1) with $m$ replaced by $m+\delta$. More precisely, we choose $\varepsilon_{1} \in(0,1)$ to ensure that

$$
(1-(m+\delta))\left(1-\varepsilon_{1}\right)=(1-m)(1-\varepsilon),
$$


namely, $\varepsilon_{1}:=\frac{\beta \varepsilon-\delta}{\beta-\delta}$. Then, by (5.32) and Proposition 5.14, there exists $\tau_{0}=$ $\tau_{0}\left(\varepsilon_{1}\right)=\tau_{0}(\varepsilon)$ such that

$$
-\mathcal{F}_{m+\delta} v(x, t) \geq 0, \quad t \geq \tau_{0},
$$

where $\mathcal{F}_{m+\delta}$ is given by $(5.25)$.

Next, by (A1) $-(\mathrm{A} 3)$, there exists $\lambda_{0}=\lambda_{0}(\delta)=\lambda_{0}(\varepsilon)>0$ such that $0 \leq v \leq$ $\lambda_{0}, v \in E$, implies

$$
0 \leq G v<\delta
$$

Clearly, (5.19) yields that $0 \leq v(x, t) \leq \lambda, x \in \mathbb{R}^{d}, t \in \mathbb{R}_{+}$. Then, by (5.31), (5.25), (5.33), (5.34) we have, for any $\lambda \in\left[0, \lambda_{0}\right]$ and for any $t \geq \tau_{0}$,

$$
-\mathcal{F} v=-\mathcal{F}_{m} v-v G v=-\mathcal{F}_{m+\delta} v+\delta v-v G v \geq 0 .
$$

The statement is proved.

Now we are ready to prove Theorem 3.3.

Proof of Theorem 3.3. Recall that, by Theorem 2.1, $0 \leq u_{0} \leq 1$ implies $0 \leq$ $u(\cdot, t) \leq 1$ for $t>0$; and then, by (A2), Gu $\leq \beta$. Rewrite (2.1) in the form (1.1) with $F$ given by (2.2), then, by (1.6), $F u \geq 0$. Therefore, for all $t>0$ and a.a. $x \in \mathbb{R}^{d}$,

$$
\begin{aligned}
u(x, t) & =e^{-t} u_{0}(x)+\int_{0}^{t} e^{-(t-s)}(a * u)(x, s) d s+\int_{0}^{t} e^{-(t-s)}(F u)(x, s) d s \\
& \geq e^{-t} u_{0}(x)+\int_{0}^{t} e^{-(t-s)}(a * u)(x, s) d s .
\end{aligned}
$$

The same inequality for $u(x, s)$ implies

$$
\begin{aligned}
u(x, t) & \geq \int_{0}^{t} e^{-(t-s)}(a * u)(x, s) d s \geq \int_{0}^{t} e^{-(t-s)} e^{-s}\left(a * u_{0}\right)(x) d s \\
& =t e^{-t}\left(a * u_{0}\right)(x) \geq t e^{-t} c(x),
\end{aligned}
$$

for all $t \geq 0$ and a.a. $x \in \mathbb{R}^{d}$, because of (3.13).

Fix an arbitrary $\varepsilon \in(0,1)$. Take any $\delta \in(0, \varepsilon)$ and consider $\lambda_{0}=\lambda_{0}(\delta)>0$ and $\tau_{0}=\tau_{0}(\delta)>\sigma$, both given by Proposition 5.17. Set now

$$
\lambda:=\min \left\{\lambda_{0}, \tau_{0} e^{-(1+(\beta-\delta \beta)) \tau_{0}}\right\} .
$$

Then, by (5.35) and (5.19), we have, for a.a. $x \in \mathbb{R}^{d}$,

$$
u\left(x, \tau_{0}\right) \geq \lambda e^{(\beta-\delta \beta) \tau_{0}} c(x) \geq \lambda \min \left\{e^{(\beta-\delta \beta) \tau_{0}} c(x), 1\right\}=g_{c, \delta, \lambda}\left(x, \tau_{0}\right) .
$$

Next, the function $g_{c, \delta, \lambda}(x, t)$ is non-decreasing in $t$, hence (5.26) yields

$$
g_{c, \delta, \lambda}(x, t) \leq v_{c, \delta, \lambda}(x, t) \leq g_{c, \delta, \lambda}(x, t+\sigma),
$$

and hence we can continue (5.36) as follows:

$$
u\left(x, \tau_{0}\right) \geq v_{c, \delta, \lambda}\left(x, \tau_{0}-\sigma\right), \quad \text { for a.a. } x \in \mathbb{R}^{d} .
$$


Therefore, by Propositions 5.17 and 5.16, one gets, for any $\tau \geq 0$ and a.a. $x \in \mathbb{R}^{d}$

$$
u\left(x, \tau_{0}+\tau\right) \geq v_{c, \delta, \lambda}\left(x, \tau_{0}-\sigma+\tau\right) \geq g_{c, \delta, \lambda}\left(x, \tau_{0}-\sigma+\tau\right),
$$

where the latter inequality is because of (5.37). As a result,

$$
u\left(x, \tau_{0}+\sigma+\tau\right) \geq \lambda \quad \text { for a.a. } x \in \Lambda\left((1-\delta)\left(\tau_{0}+\tau\right), c\right), \quad \tau \geq 0 .
$$

By Proposition 4.4, without loss of generality we may assume that $c$ is given by a strictly decreasing on $\mathbb{R}_{+}$function. We will distinguish two cases.

1. Let $c$ be given by (C1). Fix $\tau \geq 0$. Since (1.2) holds, we have that the set

$$
\begin{aligned}
\widetilde{\Lambda} & :=\left\{y \in \mathbb{R}^{d}: B_{1}(y) \subset \Lambda\left((1-\delta)\left(\tau_{0}+\tau\right), c\right)\right\} \\
& =\left\{y \in \mathbb{R}^{d}: B_{1}(y) \subset B_{\eta\left((1-\delta)\left(\tau_{0}+\tau\right), b\right)}(0)\right\}
\end{aligned}
$$

is nothing but $B_{\eta_{\delta}^{-}\left(\tau_{0}+\tau, b\right)-1}(0)$ and, moreover,

$$
\Lambda\left((1-\delta)\left(\tau_{0}+\tau\right), c\right)=\bigcup_{y \in \widetilde{\Lambda}} B_{1}(y) .
$$

Take and fix now an arbitrary $y \in \widetilde{\Lambda}$, i.e. $|y| \leq \eta\left((1-\delta)\left(\tau_{0}+\tau\right)\right)-1$. Then, by $(5.38)$,

$$
u\left(x, \tau_{0}+\sigma+\tau\right) \geq \lambda \mathbb{1}_{B_{1}(y)}(x) \text { for a.a. } x \in \mathbb{R}^{d} .
$$

Consider now equation (2.1) with the initial condition $v_{0}(x)=u\left(x, \tau_{0}+\sigma+\tau\right)$, $x \in \mathbb{R}^{d}$; let $v(x, t)$ be the corresponding solution to (2.1). By the uniqueness in Theorem 2.1, $v(x, t)=u\left(x, \tau_{0}+\sigma+\tau+t\right), t \in \mathbb{R}_{+}$.

Take an arbitrary $\mu \in(0,1)$. Apply Theorem 2.3 to the solution $v$ and $K=B_{1}(y)$; then there exists $t_{\mu} \geq 1$ such that $v(x, t) \geq \mu$ for a.a. $x \in B_{1}(y)$. As a result,

$$
u\left(x, \tau_{0}+\sigma+t_{\mu}+\tau\right) \geq \mu,
$$

for all $\tau \geq 0$ and a.a. $x \in B_{1}(y)$. Stress that $t_{\mu}$ does not depend on a $y$ with $|y| \leq \eta\left((1-\delta)\left(\tau_{0}+\tau\right)\right)-1$. As a result, by $(5.39)$ for any $\delta \in(0,1)$ and $\mu \in(0,1)$, there exist $\lambda_{0}=\lambda_{0}(\delta)>0, \tau_{0}=\tau_{0}(\delta)>0$, and $t_{\mu} \geq 1$ such that, for all $\tau \geq 0$ and for a.a. $x$ with $|x| \leq \eta\left((1-\delta)\left(\tau_{0}+\tau\right)\right)$, the inequality (5.40) holds.

By the definition of $\eta$ (see (1.2)), one gets that there exists $\tau_{1} \geq 0$ such that, for all $\tau \geq \tau_{1}$,

$$
\eta\left((1-\varepsilon)\left(\tau+\tau_{0}+\sigma+t_{\mu}\right)\right) \leq \eta\left((1-\delta)\left(\tau+\tau_{0}\right)\right),
$$

i.e. (5.40) holds for all $\tau \geq \tau_{1}$ and a.a. $x$ with $|x| \leq \eta\left((1-\varepsilon)\left(\tau+\tau_{0}+\sigma+t_{\mu}\right)\right)$. Since $\mu \in(0,1)$ was arbitrary, the latter fact yields (1.9).

2 . Let now $c$ be given by $(\mathrm{C} 2)$. Consider the norm on $\mathbb{R}^{d}$ given by

$$
|x|_{\infty}:=\left|\left(x_{1}, \ldots, x_{d}\right)\right|_{\infty}:=\max _{1 \leq j \leq d}\left|x_{j}\right| .
$$

Let $\widetilde{B}_{\frac{1}{2}}(x)$ denote the ball with the center at an $x \in \mathbb{R}^{d}$ and the radius $\frac{1}{2}$ w.r.t. the $|\cdot|_{\infty}$-norm. Then, clearly,

$$
\widetilde{B}_{\frac{1}{2}}(x)=\underset{j=1}{\stackrel{d}{X}}\left[x_{j}-\frac{1}{2}, x_{j}+\frac{1}{2}\right]=\underset{j=1}{\stackrel{X}{X}}\left[y_{j}-1, y_{j}\right]=: C_{1}(y),
$$


where $y_{j}=x_{j}+\frac{1}{2}, 1 \leq j \leq d$. For $y \in \Lambda\left((1-\delta)\left(\tau_{0}+\tau\right), c\right)$,

$$
C_{1}(y) \subset \Lambda\left((1-\delta)\left(\tau_{0}+\tau\right), c\right) .
$$

Therefore, cf. (5.39),

$$
\Lambda\left((1-\delta)\left(\tau_{0}+\tau\right), c\right)=\bigcup_{y \in \Lambda\left((1-\delta)\left(\tau_{0}+\tau\right), c\right)} C_{1}(y)
$$

Hence, one can just repeat the previous proof, applying Theorem 2.3 to the solution $v$ and $K=C_{1}(y)$ with $y \in \Lambda\left((1-\delta)\left(\tau_{0}+\tau\right), c\right)$.

\subsection{Proofs of general results}

We are going to prove the main Theorem 2.9. Consider separately proofs for the items 1(a)-(b) and 2(a)-(b).

Proof of Theorem 2.9, item 1(a). Let $\varepsilon_{0} \in(0,1)$ be chosen later. Take an arbitrary $\varepsilon \in\left(0, \varepsilon_{0}\right)$.

Let $c_{+} \in L^{1}\left(\mathbb{R}^{d}\right)$ be constructed by a long-tailed, tail-log-convex function $b_{+} \in \mathcal{D}_{d}$. Note that (2.12) yields $u_{0} \in L_{1}\left(\mathbb{R}^{d}\right)$. Therefore, one can apply Proposition 4.11 with $c=c_{+}>0$ and $f=u_{0}$; namely, there exists $D>0$ such that $a * u_{0} \geq c_{+} * u_{0} \geq D c_{+}$. Then, by Theorem 3.3, the convergence (1.9) holds, with $\varepsilon$ replaced by $\frac{\varepsilon}{2}<\varepsilon_{0}$ and $c$ replaced by $D c_{+}$. Since the functions $D b_{+}$ and $b$ are also log-equivalent, one can apply Proposition 4.7 with $b_{1}=b$ and $b_{2}=D b_{+}$, to get inclusion $\Lambda(t-\varepsilon t, c) \subset \Lambda\left(t-\frac{\varepsilon t}{2}, D c_{+}\right)$. As a result, (1.9) holds, with $c(x)=b(|x|), x \in \mathbb{R}^{d}$. Note that we had not any restrictions on $\varepsilon_{0}$ here.

Since $b^{+} \in \mathcal{D}_{d}$, we can apply Theorem 3.1 with $b_{1}=b_{2}=b^{+}$and the given $b \in \mathcal{E}_{d}$. Indeed, (B1) implies (3.3), and, for the $c_{2}$ constructed by $b^{+}$ and satisfying (C1), (2.12) is just (3.4). Therefore, (3.6) holds, that, we recall, implies (1.10) because of (3.2).

Proof of Theorem 2.9, item 1(b). The proof of (1.9) is essentially the same as that for the item 1(a), with only the difference that we will apply now Proposition 4.11 for $c=v_{\circ}>0$ and $f=a \in L^{1}\left(\mathbb{R}^{d}\right)$. Next, since $b^{\circ} \in \mathcal{D}_{d}$ and (B2) holds, we can apply Theorem 3.1 with $b_{1}=b_{2}=b^{\circ}$.

Proof of Theorem 2.9, item 2(a). Let $\varepsilon_{0} \in(0,1)$ be chosen later. Take an arbitrary $\varepsilon \in\left(0, \varepsilon_{0}\right)$. By (B1) and (2.13), we have

$$
\begin{aligned}
\left(a * u_{0}\right)(x) & \geq \zeta \int_{\mathbb{R}^{d}} b_{+}(|y|) \mathbb{1}_{\mathbb{R}_{-}^{d}}(x-y) d y \\
& =\zeta \int_{\Delta(x)} b_{+}(|y|) d y=: \tilde{c}(x), \quad x \in \mathbb{R}^{d} .
\end{aligned}
$$

One can apply Theorem 3.3 to get (1.9) with $c$ replaced by $\tilde{c}$ and $\varepsilon$ replaced by $\frac{\varepsilon}{2}$. Since the functions $b$ and $\zeta b_{+}$are log-equivalent, one can apply Proposition 4.7 with $c^{(1)}(x)=c(x):=\int_{\Delta(x)} b(|y|) d y$ and $c^{(2)}(x)=\tilde{c}(x), x \in \mathbb{R}^{d}$; and then (4.10) leads to (1.9) for this $c$.

To get (1.10) we will need just to repeat all corresponding arguments from the proof of the item 1(a) with only the difference that Theorem 3.1 will be applied now for functions satisfying $(\mathrm{C} 2)$. 
Remark 5.18. Using [32, Proposition 5.4 (Q2)] and modifying accordingly the proof of Theorem 3.3, one can replace $\mathbb{R}_{-}^{d}$ in $(2.13)$ by $\underset{j=1}{\stackrel{d}{X}}\left(-\infty, \bar{y}_{j}\right]$, for an arbitrary fixed $\bar{y} \in \mathbb{R}^{d}$.

Remark 5.19. If, additionally, $u_{0}(x)=\int_{\Delta(x)} p(y) d y, x \in \mathbb{R}^{d}$ for some $p \in$ $L^{1}\left(\mathbb{R}^{d}\right)$, then, evidently,

$$
\sup _{x \in \mathbb{R}^{d}} \frac{p(x)}{a(x)}<\infty \Longrightarrow \sup _{x \in \mathbb{R}^{d}} \frac{u_{0}(x)}{\int_{\Delta(x)} a(y) d y}<\infty .
$$

Proof of Theorem 2.9, item 2(b). First, we apply Proposition 4.11 with $f=a$ and $c$ replaced by $b_{\circ}$. Then, similarly to the proof of the item 2(a), we may apply Theorem 3.3 to get (1.9) with $c$ replaced by $v_{\circ}$ and $\varepsilon$ replaced by $\frac{\varepsilon}{2}$, and, by using the log-equivalence between $b$ and $b_{\circ}$ and Proposition 4.7, we will get (1.9) for the required $c$.

To get (1.10), one can use the same arguments as in the proof of the item 1(b).

\section{A Appendix}

Proof of Lemma 2.5. Firstly, we note that (1.7) implies (A5). Let $G$ be defined by (2.4), i.e., for $0 \leq u \in E$ and $x \in \mathbb{R}^{d}$,

$$
(G u)(x)=\beta-\alpha \frac{f(u(x))}{u(x)}-(1-\alpha) \beta\left(1-\left(a^{-} * u\right)(x)\right)^{k},
$$

where $\frac{f(s)}{s}:=\beta$ for $s=0$. Then it is straightforward to check that (A2)-(A3) and (A6) $-(\mathrm{A} 8)$ hold. We are going to prove that there exists $p \geq 0$ such that, for any $v, w \in E_{1}^{+}$with $v \leq w$,

$$
\begin{aligned}
p(w-v) & +a *(w-v) \\
& \geq(w-v) G w+v(G w-G v)+\rho \mathbb{1}_{B_{\rho}(0)} *(w-v) .
\end{aligned}
$$

Note that (Ap.1) evidently implies (A4). Next, (A10) will follow from (Ap.1) if we choose any $\delta<\rho$ with $\delta<\rho$ and any $b \in C^{\infty}\left(\mathbb{R}^{d}\right) \cap L^{\infty}\left(\mathbb{R}^{d}\right)$, such that $a-\rho \mathbb{1}_{B_{\rho}(0)} \leq b \leq a-\delta \mathbb{1}_{B_{\delta}(0)}$.

By (1.5), there exists a Lipschitz constant $K>0$, such that

$$
\begin{aligned}
G w-G v & =\alpha\left(\frac{f(v)}{v}-\frac{f(w)}{w}\right)+(1-\alpha) \beta\left(\left(1-a^{-} * v\right)^{k}-\left(1-a^{-} * w\right)^{k}\right) \\
& \leq \alpha K(w-v)+(1-\alpha) \beta k a^{-} *(w-v),
\end{aligned}
$$

where we used an elementary inequality $q^{k}-r^{k} \leq k(q-r)$ for $0 \leq r \leq q \leq 1$. Multiplying both parts of (Ap.2) on $0 \leq v \leq 1$ and using (1.7), we get

$$
v(G w-G v) \leq \alpha K(w-v)+a *(w-v)-\rho \mathbb{1}_{B_{\rho}(0)} *(w-v) .
$$

Finally, by (A2), $(w-v) G w \leq \beta(w-v)$, and therefore, the inequality (Ap.1) holds with $p:=\beta+\alpha K>0$. 
Lemma A.1. Let $\lambda>1$ and let $b: \mathbb{R}_{+} \rightarrow \mathbb{R}_{+}$be defined, for large $s$, as follows

$$
b(s)=\exp \left(-\frac{s}{(\log s)^{\lambda}}\right) .
$$

Let $\beta>0$, and define, for large $t$, the function $\eta(t):=b^{-1}\left(e^{-\beta t}\right)$. Then

$$
\eta(t) \sim \beta t(\log t)^{\lambda}, \quad t \rightarrow \infty .
$$

Proof. The equation $b(s)=e^{-\beta t}$ yields $s(\log s)^{-\lambda}=\beta t$. Making substitution $s=e^{\tau}$, one easily gets

$$
-\frac{\tau}{\lambda} e^{-\frac{\tau}{\lambda}}=-\frac{1}{\lambda(\beta t)^{\frac{1}{\lambda}}} .
$$

Since $s>e^{\lambda}$ implies $-\frac{\tau}{\lambda}<-1$ and assuming $t$ big enough, to ensure that $-\frac{1}{\lambda(\beta t)^{\frac{1}{\lambda}}}>-\frac{1}{e}$, one has that the solution to the latter equation can be given in terms of the negative real branch $W_{-1}$ of Lambert $\mathrm{W}$-function, that is the function such that $W_{-1}(\nu) \exp \left(W_{-1}(\nu)\right)=\nu, W_{-1}(\nu)<-1, \nu \in\left(-e^{-1}, 0\right)$. Namely, one gets $-\frac{\tau}{\lambda}=W_{-1}\left(-\lambda^{-1}(\beta t)^{-\frac{1}{\lambda}}\right)$, and, therefore

$$
\eta(t)=\exp \left(-\lambda W_{-1}\left(-\frac{1}{\lambda(\beta t)^{\frac{1}{\lambda}}}\right)\right) .
$$

However, $\exp \left(-W_{-1}(\nu)\right)=\nu^{-1} W_{-1}(\nu)$, therefore,

$$
\exp \left(-\lambda W_{-1}(\nu)\right)=(-\nu)^{-\lambda}\left(-W_{-1}(\nu)\right)^{\lambda},
$$

i.e.

$$
\eta(t)=\lambda^{\lambda} \beta t\left(-W_{-1}\left(-\frac{1}{\lambda(\beta t)^{\frac{1}{\lambda}}}\right)\right)^{\lambda}, \quad t>\frac{1}{\beta}\left(\frac{e}{\lambda}\right)^{\lambda} .
$$

It is well-known that $W_{-1}(\nu) \sim \log (-\nu), \nu \rightarrow 0-$. This yields (Ap.3).

Lemma A.2. Let a function $X(t) \rightarrow \infty, t \rightarrow \infty$, be such that, for large $t$,

$$
\int_{X(t)}^{\infty} \int_{X(t)}^{\infty} b(|y|) d y_{1} d y_{2}=e^{-\beta t}, \quad|y|=\sqrt{y_{1}^{2}+y_{2}^{2}}
$$

where $\beta>0$ and $b: \mathbb{R}_{+} \rightarrow \mathbb{R}_{+}$is a decreasing at $\infty$ function, such that $\int_{\mathbb{R}_{+}} b(r) r d r<\infty$. Consider the following functions

$$
c(x):=\frac{\pi}{2} \int_{\sqrt{2} x}^{\infty} b(r) r d r, \quad \mu(t):=c^{-1}\left(e^{-\beta t}\right)
$$

for large $x$ and $t$. Then, for any $\varepsilon \in(0,1)$ and large $t$,

$$
\mu(t) \geq X(t) \geq \frac{1}{2} \mu(t-\varepsilon t) .
$$

Proof. Rewriting the set $\left\{\left(y_{1}, y_{2}\right) \in \mathbb{R}^{2} \mid y_{1} \geq X(t), y_{2} \geq X(t)\right\}$ for $X(t)>0$ in polar coordinates, we obtain from (Ap.4) that, for large $t$,

$$
\begin{aligned}
e^{-\beta t} & =\int_{\sqrt{2} X(t)}^{\infty} \int_{\arcsin \frac{X(t)}{r}}^{\arccos \frac{X(t)}{r}} b(r) r d r=\int_{\sqrt{2} X(t)}^{\infty}\left(\frac{\pi}{2}-2 \arcsin \frac{X(t)}{r}\right) b(r) r d r \\
& =X(t)^{2} \int_{\sqrt{2}}^{\infty}\left(\frac{\pi}{2}-2 \arcsin \frac{1}{s}\right) b(X(t) s) s d s .
\end{aligned}
$$


Therefore, for any $\delta>0$,

$$
\begin{aligned}
c(X(t)) \geq e^{-\beta t} & \geq X(t)^{2} \int_{\sqrt{2}+\delta}^{\infty}\left(\frac{\pi}{2}-2 \arcsin \frac{1}{s}\right) b(X(t) s) s d s \\
& \geq f(\delta) X(t)^{2} \int_{\sqrt{2}+\delta}^{\infty} b(X(t) s) s d s=\frac{2}{\pi} f(\delta) c\left(\frac{\sqrt{2}+\delta}{\sqrt{2}} X(t)\right),
\end{aligned}
$$

where

$$
f(\delta):=\frac{\pi}{2}-2 \arcsin \frac{1}{\sqrt{2}+\delta} \in\left(0, \frac{\pi}{2}\right), \quad \delta>0,
$$

is an increasing function. Since $c(x)$ is decreasing, we obtain from (Ap.6) that

$$
c^{-1}\left(e^{-\beta t}\right) \geq X(t) \geq \frac{\sqrt{2}}{\sqrt{2}+\delta} c^{-1}\left(\frac{\pi}{2 f(\delta)} e^{-\beta t}\right) .
$$

Set $\lambda=\frac{\sqrt{2}}{\sqrt{2}-1}>1$. Choose $\delta>0$ such that

$$
f(\delta)=\frac{\pi}{2 \lambda}<\frac{\pi}{2}
$$

then

$$
\frac{\sqrt{2}}{\sqrt{2}+\delta}=\sqrt{2} \sin \left(\frac{\pi}{4}\left(1-\frac{1}{\lambda}\right)\right)>\frac{1}{\sqrt{2}}\left(1-\frac{1}{\lambda}\right)=\frac{1}{2},
$$

where we used the inequality $\sin x>\frac{2}{\pi} x$ for $0<x<\frac{\pi}{2}$. Then (Ap.7) implies

$$
\mu(t) \geq X(t) \geq \frac{1}{2} c^{-1}\left(\lambda e^{-\beta t}\right) .
$$

Take finally an $\varepsilon \in(0,1)$ and assume that $t$ is big enough to ensure that $e^{\varepsilon \beta t}>\lambda$. Since $c^{-1}(x)$ is a decreasing function, one gets the statement.

Remark A.3. Let (1.17)-(1.18) holds. Then, by Theorem 2.9, (1.9)-(1.10) hold with $\Lambda(t)=\Lambda(t, c)$ given by (1.11) where $c\left(x_{1}, x_{2}\right)=\int_{x_{1}}^{\infty} \int_{x_{2}}^{\infty} b(|y|) d y_{1} d y_{2}$, cf. (1.16), and $b \in \mathcal{E}_{d}$ is log-equivalent to $e^{-\sqrt{s}}, s>0$. Take $b(s)=\frac{1}{\pi} s^{-\frac{3}{2}} e^{-\sqrt{s}}$ for large $s$. By [33, Corollary 3.1], $b \in \mathcal{E}_{d}$. Let $X(t):=X_{1}(t)=X_{2}(t)$ describe the motion of the boundary of $\Lambda(t)$ in the diagonal direction in (1.16). Then, by Lemma A.2, we have, cf. (Ap.5),

$$
c(x)=\frac{\pi}{2} \frac{1}{\pi} \int_{\sqrt{2} x}^{\infty} \frac{1}{\sqrt{r}} \exp (-\sqrt{r}) d r=\exp (-\sqrt[4]{2} \sqrt{x}) .
$$

Then, by (Ap.5), $\mu(t)=\frac{\beta^{2}}{\sqrt{2}} t^{2}$. Therefore, by Lemma A.2, for any $\varepsilon \in(0,1)$ and large $t,(1.19)$ holds. 


\section{References}

[1] M. Aguerrea, C. Gomez, and S. Trofimchuk. On uniqueness of semiwavefronts. Math. Ann., 354(1):73-109, 2012.

[2] M. Alfaro and J. Coville. Propagation phenomena in monostable integrodifferential equations: Acceleration or not? J. Differential Equations, 263 (9):5727-5758, 2017.

[3] F. Andreu-Vaillo, J. M. Mazón, J. D. Rossi, and J. J. Toledo-Melero. Nonlocal diffusion problems, vol. 165 of Mathematical Surveys and Monographs. AMS Providence, RI, 2010. xvi+256 pp.

[4] D. G. Aronson. The asymptotic speed of propagation of a simple epidemic. In Nonlinear diffusion (NSF-CBMS Regional Conf. Nonlinear Diffusion Equations, Univ. Houston, Houston, Tex., 1976), pages 1-23. Res. Notes Math., No. 14. Pitman, London, 1977.

[5] H. Berestycki, J. Coville, and H.-H. Vo. Persistence criteria for populations with non-local dispersion. J. Math. Biol., 72(7):1693-1745, 2016.

[6] H. Berestycki and F. Hamel. Front propagation in periodic excitable media. Comm. Pure Appl. Math., 55(8):949-1032, 2002.

[7] H. Berestycki, G. Nadin, B. Perthame, and L. Ryzhik. The non-local Fisher-KPP equation: travelling waves and steady states. Nonlinearity, 22(12):2813--2844, 2009.

[8] B. Bolker and S. W. Pacala. Using moment equations to understand stochastically driven spatial pattern formation in ecological systems. Theor. Popul. Biol., 52(3):179-197, 1997.

[9] O. Bonnefon, J. Coville, J. Garnier, and L. Roques. Inside dynamics of solutions of integro-differential equations. Discrete and Continuous Dynamical Systems - Series B, 19(10):3057-3085, 2014.

[10] E. Bouin, J. Garnier, C. Henderson, and F. Patout. Thin front limit of an integro-differential Fisher-KPP equation with fat-tailed kernels. SIAM J. Math. Anal., 50(3):3365-3394, 2018.

[11] C. Brändle, E. Chasseigne, and R. Ferreira. Unbounded solutions of the nonlocal heat equation. Communications on Pure and Applied Analysis, 10(6):1663-1686, 2011.

[12] N. Britton. Spatial structures and periodic travelling waves in an integrodifferential reactiondiffusion population model. SIAM J. Appl. Math., 50 (6):1663--1688, 1990.

[13] X. Cabré, A.-C. Coulon, and J.-M. Roquejoffre. Propagation in FisherKPP type equations with fractional diffusion in periodic media. C. R. Math. Acad. Sci. Paris, 350(19-20):885-890, 2012.

[14] X. Cabré and J.-M. Roquejoffre. The influence of fractional diffusion in Fisher-KPP equations. Comm. Math. Phys., 320(3):679-722, 2013.

[15] A.-C. Coulon and J.-M. Roquejoffre. Transition between linear and exponential propagation in Fisher-KPP type reaction-diffusion equations. Comm. Partial Differential Equations, 37(11):2029-2049, 2012. 
[16] J. Coville, J. Dávila, and S. Martínez. Nonlocal anisotropic dispersal with monostable nonlinearity. J. Differential Equations, 244(12):3080-3118, 2008.

[17] J. Coville and L. Dupaigne. Propagation speed of travelling fronts in non local reaction-diffusion equations. Nonlinear Anal., 60(5):797-819, 2005.

[18] C. Kuehn, P. Tkachov. Pattern formation in the doubly-nonlocal FisherKPP equation. Discrete Contin. Dyn. Syst., 39(4):2077-2100, 2019.

[19] O. Diekmann. On a nonlinear integral equation arising in mathematical epidemiology. In Differential equations and applications (Proc. Third Scheveningen Conf., Scheveningen, 1977), volume 31 of North-Holland Math. Stud., pages 133-140. North-Holland, Amsterdam-New York, 1978.

[20] R. Durrett. Crabgrass, measles and gypsy moths: An introduction to modern probability. Bulletin (New Series) of the American Mathematical Society, 18(2):117-143, 1988.

[21] L. C. Evans and P. E. Souganidis. A PDE approach to geometric optics for certain semilinear parabolic equations. Indiana Univ. Math. J., 38(1): 141-172, 1989.

[22] S. Gourley, M. Chaplain, and F. Davidson. Spatio-temporal pattern formation in a nonlocal reaction-diffusion equation. Dynamical Systems, 16 (2):173-192, 2001.

[23] G. Faye and M. Holzer. Modulated traveling fronts for a nonlocal FisherKPP equation: A dynamical systems approach. J. Differential Equations, 258(7):2257-2289, 2015.

[24] P. Felmer and M. Yangari. Fast propagation for fractional KPP equations with slowly decaying initial conditions. SIAM J. Math. Anal., 45(2):662678, 2013.

[25] P. C. Fife. Mathematical aspects of reacting and diffusing systems, volume 28 of Lecture Notes in Biomathematics. Springer-Verlag, Berlin-New York, 1979. iv+185 pp.

[26] D. Finkelshtein, Y. Kondratiev, Y. Kozitsky, and O. Kutoviy. The statistical dynamics of a spatial logistic model and the related kinetic equation. Math. Models Methods Appl. Sci., 25(2):343-370, 2015.

[27] D. Finkelshtein, Y. Kondratiev, and O. Kutoviy. Semigroup approach to birth-and-death stochastic dynamics in continuum. J. Funct. Anal., 262 (3):1274-1308, 2012.

[28] D. Finkelshtein, Y. Kondratiev, and P. Tkachov. Existence and properties of traveling waves for doubly nonlocal Fisher-KPP equations. Electronic J. Differential Equations, 2019(10):1-27, 2019.

[29] D. Finkelshtein, Y. Kondratiev, and P. Tkachov. Doubly nonlocal FisherKPP equation: Speeds and uniqueness of traveling waves. J. Math. Anal. Appl., 475(1):94-122, 2019.

[30] D. Finkelshtein, Y. Kondratiev, and P. Tkachov. Doubly nonlocal Fisher-KPP equation: Front propagation. Applicable Analysis, doi: 10.1080/00036811.2019.1643011, 2019. 
[31] D. Finkelshtein and P. Tkachov. Accelerated nonlocal nonsymmetric dispersion for monostable equations on the real line. Applicable Analysis, 98 (4):756-780, 2019.

[32] D. Finkelshtein and P. Tkachov. The hair-trigger effect for a class of nonlocal nonlinear equations. Nonlinearity, 31(6):2442-2479, 2018.

[33] D. Finkelshtein and P. Tkachov. Kesten's bound for sub-exponential densities on the real line and its multi-dimensional analogues. Advances in Applied Probability, 50(2): 373-395, 2018.

[34] R. Fisher. The wave of advance of advantageous genes. Ann. Eugenics, 7: 335-369, 1937.

[35] S. Foss, D. Korshunov, and S. Zachary. An introduction to heavy-tailed and subexponential distributions. Springer Series in Operations Research and Financial Engineering. Springer, New York, second edition, 2013. xii +157 pp.

[36] N. Fournier and S. Méléard. A microscopic probabilistic description of a locally regulated population and macroscopic approximations. The Annals of Applied Probability, 14(4):1880-1919, 2004.

[37] J. Garnier. Accelerating solutions in integro-differential equations. SIAM Journal on Mathematical Analysis, 43(4):1955-1974, 2011.

[38] O. Hallatschek and D. S. Fisher. Acceleration of evolutionary spread by long-range dispersal. Proceedings of the National Academy of Sciences, 111 (46):E4911-E4919, 2014

[39] F. Hamel, J. Garnier, and L. Roques. Transition fronts and stretching phenomena for a general class of reaction-dispersion equations. Disc. Cont. Dyn. Syst. - Ser. A, 37(2):743-756, 2017.

[40] F. Hamel and L. Roques. Fast propagation for KPP equations with slowly decaying initial conditions. J. Differential Equations, 249(7):1726-1745, Oct. 2010 .

[41] C. Henderson. Propagation of solutions to the Fisher-KPP equation with slowly decaying initial data. Nonlinearity, 29(11):3215, 2016.

[42] D. Henry. Geometric theory of semilinear parabolic equations, volume 840 of Lecture Notes in Mathematics. Springer-Verlag, Berlin-New York, 1981. iv $+348 \mathrm{pp}$.

[43] A. N. Kolmogorov, I. G. Petrovsky, and N. S. Piskunov. Étude de l'équation de la diffusion avec croissance de la quantité de matière et son application à un problème biologique. Bull. Univ. État Moscou Sér. Inter. A, 1:1-26, 1937.

[44] Y. Kondratiev, S. Molchanov, A. Piatnitski, and E. Zhizhina. Resolvent bounds for jump generators. Applicable Analysis, 2016. doi: 10.1080/00036811.2016.1263838.

[45] M. A. Lewis, S. V. Petrovskii, and J. R. Potts. The mathematics behind biological invasions, volume 44 of Interdisciplinary Applied Mathematics. Springer, [Cham], 2016. xvi+362 pp. With a foreword by James D. Murray. 
[46] B. R. Liu and M. Kot. Accelerating invasions and the asymptotics of fattailed dispersal. J. Theoretical Biology, 471:22-41, 2019.

[47] J. Medlock and M. Kot. Spreading disease: integro-differential equations old and new. Mathematical Biosciences, 184(2):201-222, Aug. 2003.

[48] S. Méléard and S. Mirrahimi. Singular limits for reaction-diffusion equations with fractional Laplacian and local or nonlocal nonlinearity. Comm. Partial Differential Equations, 40(5):957-993, 2015.

[49] D. Mollison. Possible velocities for a simple epidemic. Advances in Appl. Probability, 4:233-257, 1972.

[50] D. Mollison. The rate of spatial propagation of simple epidemics. In Proceedings of the Sixth Berkeley Symposium on Mathematical Statistics and Probability (Univ. California, Berkeley, Calif., 1970/1971), Vol. III: Probability theory, pages 579-614. Univ. California Press, Berkeley, Calif., 1972.

[51] J. D. Murray. Mathematical biology. II, volume 18 of Interdisciplinary Applied Mathematics. Springer-Verlag, New York, third edition, 2003. xxvi+811 pp. Spatial models and biomedical applications.

[52] B. Perthame and P. E. Souganidis. Front propagation for a jump process model arising in spatial ecology. Discrete Contin. Dyn. Syst., 13(5):12351246, 2005.

[53] K. Schumacher. Travelling-front solutions for integro-differential equations. I. J. Reine Angew. Math., 316:54-70, 1980.

[54] W. Shen and A. Zhang. Spreading speeds for monostable equations with nonlocal dispersal in space periodic habitats. J. Differential Equations, 249 (4):747-795, Aug. 2010.

[55] Y.-J. Sun, W.-T. Li, and Z.-C. Wang. Traveling waves for a nonlocal anisotropic dispersal equation with monostable nonlinearity. Nonlinear Anal., 74(3):814-826, 2011.

[56] H. Weinberger. On sufficient conditions for a linearly determinate spreading speed. Discrete and Continuous Dynamical Systems - Series B, 17(6):22672280, 2012.

[57] H. F. Weinberger. Asymptotic behavior of a model in population genetics. pages 47-96. Lecture Notes in Math., Vol. 648, 1978.

[58] W.-B. Xu, W.-T. Li, S. Ruan. Fast propagation for reaction-diffusion cooperative systems. J. Differential Equations, 265(2):645-670, 2018.

[59] H. Yagisita. Existence and nonexistence of traveling waves for a nonlocal monostable equation. Publ. Res. Inst. Math. Sci., 45(4):925-953, 2009.

[60] G.-B. Zhang, W.-T. Li, and Z.-C. Wang. Spreading speeds and traveling waves for nonlocal dispersal equations with degenerate monostable nonlinearity. J. Differential Equations, 252(9):5096-5124, 2012. 